\title{
Abnormal Solvent Effects on Hydrogen Atom Abstraction. 2. Resolution of the Curcumin Antioxidant Controversy. The Role of Sequential Proton Loss Electron Transfer (SPLET)
}

Grzegorz Litwinienko and K. U. Ingold

National Research Council, 100 Sussex Drive, Ottawa, Ontario, Canada, K1A 0R6

\section{SUPPORTING MATERIAL}

TABLE OF CONTENTS

Title

page

Table S1. Kinetic data for the reaction of dpph $^{\bullet}$ with $\mathbf{C U}$ in neat and acidified 1,4-dioxane.

Table S2. Kinetic data for the reaction of $\mathbf{d p p h}^{\bullet}$ with $\mathbf{D H Z}$ in neat and acidified 1,4-dioxane

S-3

Table S3. Kinetic data for the reaction of $\mathbf{d p p h}^{\bullet}$ with IE in neat and acidified 1,4-dioxane.

Table S4. Kinetic data for the reaction of $\mathrm{dpph}^{\bullet}$ with $\mathrm{CU}$ in methanol.

S-4

Table S5. Kinetic data for the reaction of $\mathrm{dpph}^{\bullet}$ with $\mathrm{CU}$ in acidified methanol.

Table S6. Kinetic data for the reaction of $\mathrm{dpph}^{\bullet}$ with $\mathrm{DHZ}$ in methanol and acidified methanol.

Table S7. Kinetic data for the reaction of $\mathrm{dpph}^{\bullet}$ with IE in pure methanol.

Table S8. Kinetic data for the reaction of $\mathbf{d p p h}^{\bullet}$ with IE in acidified methanol.

Table S9. Kinetic data for the reaction of $\mathrm{dpph}^{\bullet}$ with AcAc in methanol.

Table S10. Kinetic data for the reaction of $\mathrm{dpph}^{\bullet}$ with $\mathbf{C U}$ in neat ethanol and acidified ethanol.

Table S11. Kinetic data for the reaction of $\mathrm{dpph}^{\bullet}$ with $\mathrm{DHZ}$ in ethanol.

Table S12. Kinetic data for the reaction of $\mathrm{dpph}^{\bullet}$ with $\mathrm{DHZ}$ in acidified ethanol.

Table S13. Kinetic data for the reaction of dpph $^{\bullet}$ with IE in neat ethanol and acidified ethanol.

Table S14. Kinetic data for the reaction of $\mathbf{d p p h}^{\bullet}$ with AcAc in dioxane.

Table S15. Kinetic data for the reaction of $\mathrm{dpph}^{\bullet}$ with $\mathbf{C U}$ in neat and acidified ethyl acetate.

Table S16. Kinetic data for the reaction of dpph $^{\bullet}$ with $\mathrm{DHZ}$ in neat and acidified ethyl acetate.

Table S17. Kinetic data for the reaction of $\mathbf{d p p h}^{\bullet}$ with IE in neat and acidified ethyl acetate.

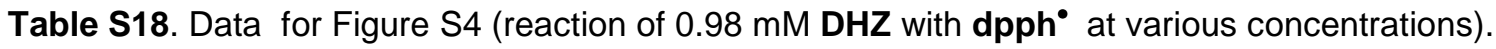

Table S19. Kinetic data for the reaction of $\mathrm{dpph}^{\bullet}$ with $\mathrm{DHZ}$, 2-MeO-phenol, IE, and 4-Me-2-MeO-phenol in heptane.

Table S20. Kinetic data for the reaction of $\mathrm{dpph}^{\bullet}$ with 2-MeO-phenol and 4-Me-2-MeO-phenol in in ethyl acetate.

Table S21. Kinetic data for the reaction of dpph $^{\bullet}$ with 2-MeO-phenol and 4-Me-2-MeO-phenol in dioxane. 


\section{TABLE OF CONTENTS continued...}

Title

Page

Table S22. Kinetic data for the reaction of $\mathbf{d p p h}^{\bullet}$ with acetylacetone in dioxane 24 hours after preparation S-25 of AcAc / dioxane solutions.

Table S23. Kinetic data for the reaction of dpph $^{\bullet}$ with AcAc in methanol freshly prepared and 24 hours after preparation of AcAc / methanol solutions

Table S24. Parameters used for calculation of the equilibrium constant $K_{\text {ArOH/S }}^{S}$ for HB complex formation between DMSO and four phenols.

Table S25. Values $K_{A}^{H_{i}}, \log K_{A}^{H_{i}}$, and $\alpha_{2}^{H}$ for studied ortho-methoxyphenols calculated from IR measurements of HB complex formation with DMSO.

Table S26. Parameters used for calculation of the equilibrium constant $K_{\text {ArOH/S }}^{S}$ for $\mathrm{HB}$ complex formation between 1,4-dioxane and 4-F-phenol and 2-OMe-phenol.

Table S27. Determination of ionization constant of 2-MeO-phenol.

Table S28. Determination of ionization constant of 2-MeO-phenol.

Table S29. Determination of ionization constant of 4-Me-2-MeO-phenol

Table S30. Determination of ionization constant of 4-Me-2-MeO-phenol.

Table S31. Determination of ionization constant of IE.

Table S32. Determination of ionization constant of IE.

Table S33. Determination of ionization constant of DHZ.

Table S34. Determination of ionization constant of DHZ.

Table S35. Determination of ionization constant of DHZ.

Table S36. Experimental and literature values of $\mathrm{p} K_{\mathrm{a}}$ for the studied phenols.

Figure S1. Dependence of the $\log k^{s}$ vs. $\left[\mathrm{CH}_{3} \mathrm{CO}_{2} \mathrm{H}\right]$ for $\mathrm{CU}+\mathrm{dpph}^{\bullet}$ in methanol.

Figure S2. Dependence of the $k_{e x}$ vs. concentration of [AcAc] in methanol.

Figure S3. Examples of the dpph ${ }^{\bullet}$ decay traces for fixed initial concentration of $\mathbf{D H Z}$ and variable initial concentration of $\mathbf{d p p h}^{\bullet}$ in methanol.

Figure S4 A: Decay traces of dpph $^{\bullet}$ reacting with $\mathbf{D H Z}$ in methanol, [DHZ] $=0.98 \mathrm{mM}=$ const.

Figure S4 B: Plot of [dpph $\left.{ }^{\circ}\right]_{0}-\left[\mathbf{d p p h}^{\circ}\right]_{\text {intercept }}=\Delta\left[\mathbf{d p p h}^{\circ}\right]$ as a function of reciprocal of [dpph $]_{0}$.

Figure S5. IR spectra of IE in CCl4 containing various concentrations of DMSO.

Figure S6. IR spectra of $\mathrm{DHZ}$ in $\mathrm{CCl}_{4}$ containing various concentrations of DMSO

Figure S7. Plots of $[\mathrm{ArOH}]_{0} /[\mathrm{ArOH}]_{\text {free }}$ vs. $[\mathrm{DMSO}]_{\text {free }}$ for phenols listed in Table S24.

Figure S8. Plots of $[\mathrm{ArOH}]_{0} /[\mathrm{ArOH}]_{\text {free }}$ vs. [dioxane $]_{\text {free }}$ for phenols listed in Table S26. 
Table S1. Kinetic data for the reaction of $\mathbf{d p p h}^{\bullet}$ with curcumin in 1,4-dioxane and in 1,4-dioxane containing $10 \mathrm{mM} \mathrm{CH}_{3} \mathrm{CO}_{2} \mathrm{H}$. Concentration [CU], pseudo-first-order rate constant $\mathrm{k}_{\mathrm{ex}}, \mathrm{R}^{2}$ for each data set The calculated mean bimolecular rate constant is denoted as $\left(\boldsymbol{k}^{\boldsymbol{S}} \pm\right.$ absolute error). In all tables abbreviations for phenol names are the same as in main text: curcumin (CU), dehydrozingerone (DHZ), and isoeugenol (IE).

\begin{tabular}{|c|c|c|c|c|c|}
\hline \multicolumn{2}{|c|}{ neat dioxane } & \multicolumn{2}{|c|}{ neat dioxane } & \multicolumn{2}{|c|}{$10 \mathrm{mM} \mathrm{CH}_{3} \mathrm{CO}_{2} \mathrm{H}$} \\
\hline $\begin{array}{l}{[\mathrm{CU}]} \\
/ \mathrm{mM}\end{array}$ & $\begin{array}{l}\mathrm{k}_{\mathrm{ex}} \times 10^{3} \\
/ \mathrm{s}-1\end{array}$ & $\begin{array}{l}{[\mathrm{CU}]} \\
/ \mathrm{mM}\end{array}$ & $\begin{array}{c}\mathrm{k}_{\mathrm{ex}} \times 10^{3} \\
/ \mathrm{s}-1\end{array}$ & $\begin{array}{l}{[\mathrm{CU}]} \\
/ \mathrm{mM}\end{array}$ & $\begin{array}{c}\mathrm{k}_{\mathrm{ex}} \times 10^{3} \\
/ \mathrm{s}-1\end{array}$ \\
\hline 0.700 & 1.00 & 0.739 & 1.18 & 0.831 & 1.25 \\
\hline 0.545 & 0.810 & 0.575 & 0.910 & 0.647 & 0.912 \\
\hline 0.409 & 0.621 & 0.431 & 0.649 & 0.485 & 0.772 \\
\hline 0.292 & 0.444 & 0.308 & 0.507 & 0.346 & 0.550 \\
\hline 0.195 & 0.318 & 0.205 & 0.368 & 0.231 & 0.443 \\
\hline 0.117 & 0.180 & 0.123 & 0.236 & 0.139 & 0.245 \\
\hline 0.058 & 0.107 & 0.062 & 0.154 & 0.069 & 0.182 \\
\hline \multicolumn{2}{|c|}{ 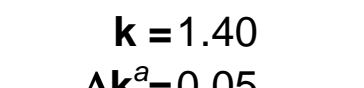 } & \multicolumn{2}{|c|}{$\begin{aligned} \Delta \mathbf{k}^{a} & =0.07 \\
\mathbf{R}^{2} & =0.9966\end{aligned}$} & \multicolumn{2}{|c|}{$\begin{aligned} \Delta \mathbf{k}^{a} & =0.11 \\
\mathbf{R}^{2} & =0.9916\end{aligned}$} \\
\hline
\end{tabular}

${ }^{a}$ calculated as the confidence interval of the slope for the $90 \%$ confidence level.

$k^{S}=1.4 \pm 0.1 \mathrm{M}^{-1} \mathrm{~s}^{-1}$

$k^{S}{ }_{10 \mathrm{mM} \mathrm{CH}} \mathrm{COO}_{\mathrm{H}}=1.4 \pm 0.1 \mathrm{M}^{-1} \mathrm{~s}^{-1}$

Table S2. Kinetic data for the reaction of $\mathbf{d p p h}^{\bullet}$ with $\mathrm{DHZ}$ in dioxane. Symbols are the same as described in Table $\mathbf{S 1 .}$

\begin{tabular}{cccccc}
\hline \multicolumn{2}{c}{ neat dioxane } & \multicolumn{2}{c}{ neat dioxane } & \multicolumn{2}{c}{$10 \mathrm{mM} \mathrm{CH}_{3} \mathrm{CO}_{2} \mathrm{H}$} \\
\hline $\begin{array}{c}\mathrm{k}_{\mathrm{ex}} \times 10^{3} \\
/ \mathrm{sH} \text {-1 }\end{array}$ & $\begin{array}{c}\mathrm{C} \\
/ \mathrm{mM}\end{array}$ & $\begin{array}{c}\mathrm{k}_{\mathrm{ex} \times 10^{3}} \\
/ \mathrm{s}-1\end{array}$ & $\begin{array}{c}\mathrm{C} \\
/ \mathrm{mM}\end{array}$ & $\begin{array}{c}\mathrm{k}_{\mathrm{ex}} \times 10^{3} \\
/ \mathrm{s}-1\end{array}$ \\
\hline 2.82 & 0.94 & 2.17 & 0.72 & 1.66 & 0.53 \\
2.20 & 0.77 & 1.69 & 0.53 & 1.29 & 0.40 \\
1.71 & 0.62 & 1.31 & 0.40 & 0.97 & 0.31 \\
1.28 & 0.46 & 0.99 & 0.32 & 0.73 & 0.25 \\
0.96 & 0.33 & 0.74 & 0.24 & 0.55 & 0.17 \\
0.72 & 0.26 & 0.55 & 0.17 & & \\
0.54 & 0.21 & 0.42 & 0.14 & &
\end{tabular}

\begin{tabular}{|c|c|c|}
\hline $\begin{aligned} \mathbf{k} & =0.33 \mathrm{M}^{-1} \mathrm{~s}^{-1} \\
\Delta \mathbf{k}^{\mathrm{a}} & =0.02 \mathrm{M}^{-1} \mathrm{~s}^{-1} \\
\mathbf{R}^{2} & =0.9961\end{aligned}$ & $\begin{aligned} \mathbf{k} & =0.33 \mathrm{M}^{-1} \mathrm{~s}^{-1} \\
\Delta \mathbf{k}^{a} & =0.02 \\
\mathbf{R}^{2} & =0.9964\end{aligned}$ & $\begin{aligned} \mathbf{k} & =0.31 \mathrm{M}^{-1} \mathrm{~s}^{-1} \\
\Delta \mathbf{k}^{\mathbf{a}} & =0.03 \\
\mathbf{R}^{2} & =0.9950\end{aligned}$ \\
\hline
\end{tabular}

${ }^{a}$ calculated as the confidence interval of the slope for the $90 \%$ confidence level.

$k^{S}=0.33 \pm 0.02 \mathrm{M}^{-1} \mathrm{~s}^{-1}$

$k^{S}{ }_{10 \mathrm{mM} \mathrm{CH} 3 \mathrm{CO} 2 \mathrm{H}}=0.31 \pm 0.03 \mathrm{M}^{-1} \mathrm{~s}^{-1}$ 
Table S3. Kinetic data for the reaction of $\mathbf{d p p h}^{\bullet}$ with IE (IE) in 1,4-dioxane (neat and acidified). Symbols are the same as described in Table S1.

\begin{tabular}{|c|c|c|c|c|c|}
\hline \multicolumn{2}{|c|}{ neat dioxane } & \multicolumn{2}{|c|}{ neat dioxane } & \multicolumn{2}{|c|}{$10 \mathrm{mM} \mathrm{CH}_{3} \mathrm{CO}_{2} \mathrm{H}$} \\
\hline $\begin{array}{l}{[\mathrm{IE}]} \\
/ \mathrm{mM}\end{array}$ & $\begin{array}{c}\mathrm{k}_{\mathrm{ex}} \times 10^{3} \\
/ \mathrm{s}-1\end{array}$ & $\begin{array}{l}{[\mathrm{IE}]} \\
/ \mathrm{mM}\end{array}$ & $\begin{array}{c}\mathrm{k}_{\mathrm{ex}} \times 10^{3} \\
/ \mathrm{s}-1\end{array}$ & $\begin{array}{l}{[\mathrm{IE}]} \\
/ \mathrm{mM}\end{array}$ & $\begin{array}{c}\mathrm{k}_{\mathrm{ex}} \times 10^{3} \\
/ \mathrm{s}-1\end{array}$ \\
\hline 8.90 & 20.85 & 8.90 & 21.35 & 19.46 & 44.39 \\
\hline 6.92 & 15.77 & 6.68 & 16.16 & 15.13 & 36.44 \\
\hline 5.19 & 11.92 & 5.01 & 12.13 & 11.77 & 27.32 \\
\hline 3.71 & 8.48 & 3.76 & 9.22 & 8.83 & 20.87 \\
\hline 2.47 & 5.96 & 2.50 & 6.22 & 6.31 & 15.09 \\
\hline 1.48 & 3.62 & 1.50 & 3.85 & 4.20 & 9.92 \\
\hline 0.89 & 2.26 & 0.90 & 2.46 & 2.52 & 6.16 \\
\hline 0.53 & 1.43 & 0.54 & 1.53 & & \\
\hline 0.32 & $\begin{array}{l}0.91 \\
.3 \mathrm{M}^{-1} \mathrm{~s}^{-1} \\
.04 \\
.9994\end{array}$ & & $\begin{array}{l}0.92 \\
4 \mathrm{M}^{-1} \mathrm{~s}^{-1} \\
01 \\
9999\end{array}$ & $\begin{array}{r}\mathbf{k}= \\
\Delta \mathbf{k}^{a}= \\
\mathbf{R}^{2}=\end{array}$ & $\begin{array}{l}3 M^{-1} s^{-1} \\
981\end{array}$ \\
\hline
\end{tabular}

${ }^{a}$ calculated as the confidence interval of the slope for the $90 \%$ confidence level.

$k^{S}=2.4 \pm 0.1 \mathrm{M}^{-1} \mathrm{~s}^{-1}$

$k^{S}{ }_{10 \mathrm{MM} \mathrm{CH} 3 \mathrm{CO}_{2 H}}=2.3 \pm 0.1 \mathrm{M}^{-1} \mathrm{~s}^{-1}$

Table S4. Kinetic data for the reaction of $\mathrm{dpph}^{\bullet}$ with $\mathrm{CU}$ in methanol. Symbols are the same as described in Table S1.

\begin{tabular}{cccc}
\hline $\mathrm{CU}] \times 10^{5}$ & $\mathrm{k}_{\mathrm{ex}}$ & $\begin{array}{c}{[\mathrm{CU}] \times 10^{5}} \\
/ \mathrm{M}\end{array}$ & $\begin{array}{c}\mathrm{k}_{\mathrm{ex}} \\
/ \mathrm{s}-1\end{array}$ \\
\hline 24.4 & $/ \mathrm{s}-1$ & 35.6 & 5.65 \\
16.3 & 4.16 & 23.7 & 3.05 \\
10.8 & 3.30 & 15.8 & 2.33 \\
7.2 & 1.97 & 10.5 & 1.14 \\
4.8 & 1.59 & 7.02 & 1.01 \\
3.2 & 1.11 & 4.68 & 0.723 \\
2.1 & 0.679 & 3.12 & 0.450
\end{tabular}

\begin{tabular}{|c|c|}
\hline $\begin{aligned} \mathbf{k} & =17.3 \times 10^{3} \mathrm{M}^{-1} \mathrm{~s}^{-1} \\
\Delta \mathbf{k}^{a} & =2.1 \times 10^{3}\end{aligned}$ & $\begin{aligned} \mathbf{k} & =15.5 \times 10^{3} \mathrm{M}^{-1} \mathrm{~s}^{-1} \\
\Delta \mathbf{k}^{a} & =2.0 \times 10^{3}\end{aligned}$ \\
\hline $\mathbf{R}^{2}=0.9891$ & $\mathbf{R}^{2}=0.9771$ \\
\hline
\end{tabular}


Table S5. Kinetic data for the reaction of $\mathrm{dpph}^{\bullet}$ with $\mathrm{CU}$ in acidified methanol. Symbols are the same as described in Table S1.

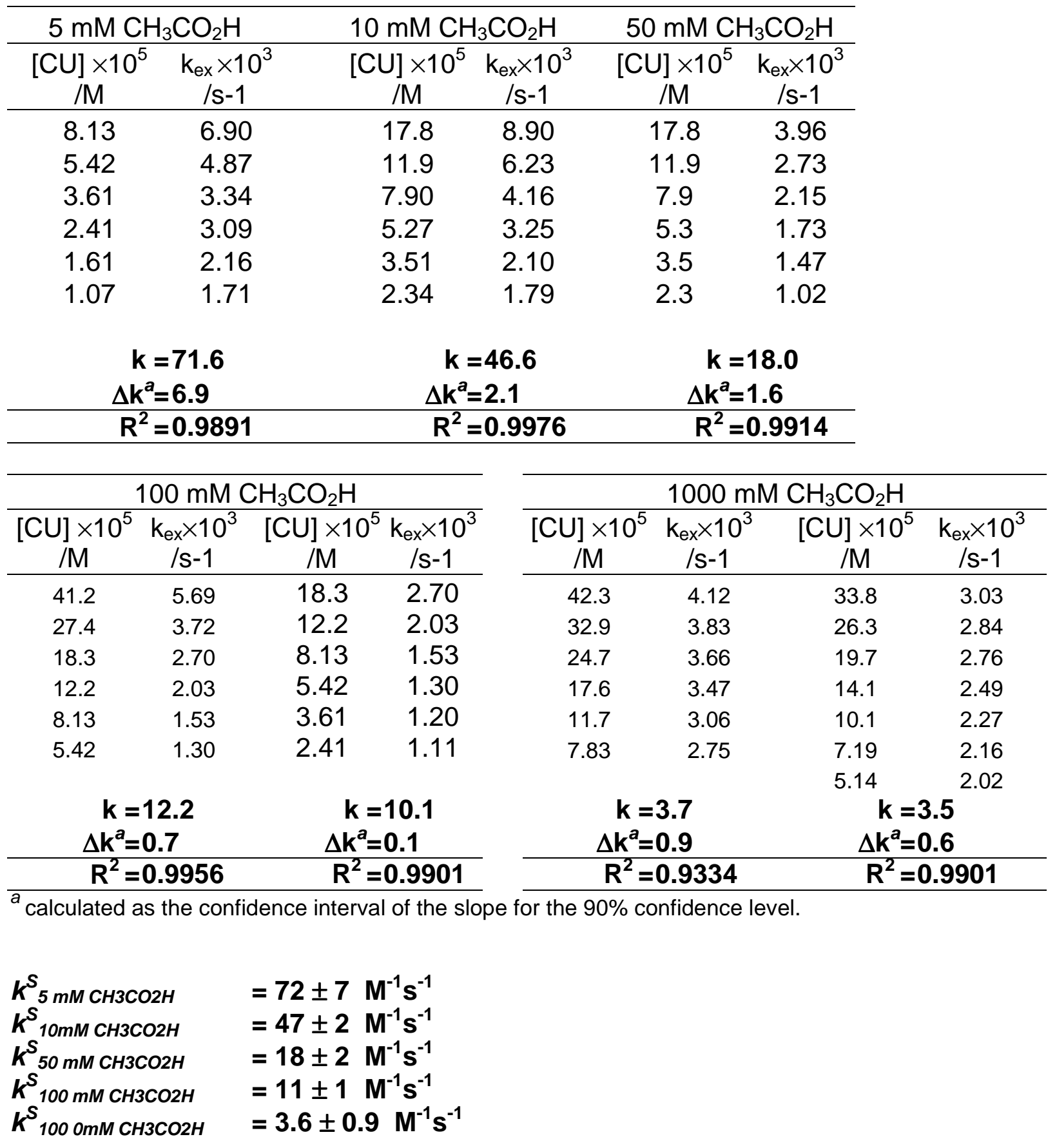


Table S6. Kinetic data for the reaction of $\mathbf{d p p h}^{\bullet}$ with $\mathrm{DHZ}$ in methanol and acidified methanol. Symbols are the same as described in Table S1.

\begin{tabular}{|c|c|c|c|c|c|}
\hline \multicolumn{2}{|c|}{ neat $\mathrm{MeOH}$} & \multicolumn{2}{|c|}{ neat $\mathrm{MeOH}$} & \multicolumn{2}{|c|}{ neat $\mathrm{MeOH}$} \\
\hline $\begin{array}{c}\text { [DHZ] } \\
/ \mathrm{mM}\end{array}$ & $\begin{array}{c}k_{e x} \\
/ s-1\end{array}$ & $\begin{array}{c}{[\mathrm{DHZ}]} \\
/ \mathrm{mM}\end{array}$ & $\begin{array}{l}\mathrm{k}_{\mathrm{ex}} \\
/ \mathrm{s}-1\end{array}$ & $\begin{array}{c}\text { [DHZ] } \\
/ \mathrm{mM}\end{array}$ & $\begin{array}{c}\mathrm{k}_{\mathrm{ex}} \\
/ \mathrm{s}-1\end{array}$ \\
\hline 1.04 & 1.79 & 1.62 & 2.02 & 0.939 & 1.48 \\
\hline 0.812 & 1.59 & 1.26 & 1.85 & 0.730 & 1.33 \\
\hline 0.609 & 1.09 & 0.946 & 1.51 & 0.548 & 0.978 \\
\hline 0.435 & 0.819 & 0.710 & 1.33 & 0.391 & 0.778 \\
\hline 0.174 & 0.549 & 0.532 & 0.946 & 0.261 & 0.495 \\
\hline 0.104 & 0.410 & 0.355 & 0.563 & 0.157 & 0.497 \\
\hline 0.063 & 0.300 & 0.237 & 0.236 & 0.056 & 0.233 \\
\hline 0.038 & 0.261 & 0.158 & 0.159 & 0.034 & 0.169 \\
\hline \multicolumn{2}{|c|}{$\begin{aligned} \mathbf{k} & =1536 \mathrm{M}^{-1} \mathrm{~s}^{-1} \\
\Delta \mathbf{k}^{a} & =130 \\
\mathbf{R}^{2} & =0.9784\end{aligned}$} & \multicolumn{2}{|c|}{$\begin{aligned} \mathbf{k} & =1332 \mathrm{M}^{-1} \mathrm{~s}^{-1} \\
\Delta \mathbf{k}^{a} & =270 \\
\mathbf{R}^{2} & =0.9333\end{aligned}$} & \multicolumn{2}{|c|}{$\begin{aligned} \mathbf{k} & =1470 \mathrm{M}^{-1} \mathrm{~S}^{-1} \\
\Delta \mathbf{k}^{\mathbf{a}} & =150 \\
\mathbf{R}^{2} & =0.9823\end{aligned}$} \\
\hline
\end{tabular}

\begin{tabular}{|c|c|c|c|c|c|c|c|}
\hline \multicolumn{4}{|c|}{$5 \mathrm{mM} \mathrm{CH}_{3} \mathrm{CO}_{2} \mathrm{H}$} & \multicolumn{4}{|c|}{$10 \mathrm{mM} \mathrm{CH}_{3} \mathrm{CO}_{2} \mathrm{H}$} \\
\hline $\begin{array}{c}{[\mathrm{DHZ}]} \\
/ \mathrm{mM}\end{array}$ & $\begin{array}{c}\mathrm{k}_{\mathrm{ex}} \times 10^{3} \\
/ \mathrm{s}-1\end{array}$ & $\begin{array}{c}\text { [DHZ] } \\
/ \mathrm{mM}\end{array}$ & $\begin{array}{c}\mathrm{k}_{\mathrm{ex}} \times 10^{3} \\
/ \mathrm{s}-1\end{array}$ & $\begin{array}{l}\text { [DHZ] } \\
/ \mathrm{mM}\end{array}$ & $\begin{array}{c}\mathrm{k}_{\mathrm{ex}} \times 10^{3} \\
/ \mathrm{s}-1\end{array}$ & $\begin{array}{c}\text { [DHZ] } \\
/ \mathrm{mM}\end{array}$ & $\begin{array}{c}\mathrm{k}_{\mathrm{ex}} \times 10^{3} \\
/ \mathrm{s}-1\end{array}$ \\
\hline 1.65 & 17.4 & 2.26 & 25.5 & 2.07 & 13.5 & 1.59 & 11.1 \\
\hline 1.29 & 14.7 & 1.75 & 21.1 & 1.61 & 10.1 & 1.24 & 8.91 \\
\hline 0.965 & 12.0 & 1.32 & 16.8 & 1.21 & 8.15 & 0.928 & 7.46 \\
\hline 0.689 & 9.33 & 0.940 & 12.9 & 0.862 & 6.78 & 0.696 & 5.49 \\
\hline 0.459 & 6.72 & 0.627 & 9.38 & 0.574 & 5.02 & 0.522 & 4.25 \\
\hline 0.276 & 4.54 & 0.376 & 6.07 & 0.345 & 3.45 & 0.348 & 3.18 \\
\hline 0.138 & 2.73 & 0.188 & 3.74 & 0.172 & 2.11 & 0.232 & 2.33 \\
\hline 0.069 & 1.95 & 0.094 & 2.23 & & & & \\
\hline \multicolumn{2}{|c|}{$\begin{aligned} \mathbf{k} & =9.9 \mathrm{M}^{-1} \mathrm{~s}^{-1} \\
\Delta \mathbf{k}^{\mathbf{a}} & =0.7 \\
\mathbf{R}^{2} & =0.9908\end{aligned}$} & \multicolumn{2}{|c|}{$\begin{aligned} \mathbf{k} & =10.1 \mathrm{M}^{-1} \mathrm{~s}^{-1} \\
\Delta \mathbf{k}^{\mathbf{a}} & =0.6 \\
\mathbf{R}^{2} & =0.9941\end{aligned}$} & \multicolumn{2}{|c|}{$\begin{aligned} \mathbf{k} & =5.7 \mathrm{M}^{-1} \mathrm{~s}^{-1} \\
\Delta \mathbf{k}^{\mathbf{a}} & =0.4 \\
\mathbf{R}^{2} & =0.9919\end{aligned}$} & \multicolumn{2}{|c|}{$\begin{aligned} \mathbf{k} & =6.5 \mathrm{M}^{-1} \mathrm{~s}^{-1} \\
\Delta \mathbf{k}^{\mathbf{a}} & =0.4 \\
\mathbf{R}^{2} & =0.9953\end{aligned}$} \\
\hline
\end{tabular}


Table S6. continued

\begin{tabular}{|c|c|c|c|c|c|}
\hline \multicolumn{4}{|c|}{$50 \mathrm{mM} \mathrm{CH}_{3} \mathrm{CO}_{2} \mathrm{H}$} & \multicolumn{2}{|c|}{$100 \mathrm{mM} \mathrm{CH}_{3} \mathrm{CO}_{2} \mathrm{H}$} \\
\hline $\begin{array}{c}{[\mathrm{DHZ}]} \\
/ \mathrm{mM}\end{array}$ & $\begin{array}{c}\mathrm{k}_{\mathrm{ex}} \times 10^{3} \\
/ \mathrm{s}-1\end{array}$ & $\begin{array}{c}{[\mathrm{DHZ}]} \\
/ \mathrm{mM}\end{array}$ & $\begin{array}{c}\mathrm{k}_{\mathrm{ex}} \times 10^{3} \\
/ \mathrm{s}-1\end{array}$ & $\begin{array}{c}{[\mathrm{DHZ}]} \\
/ \mathrm{mM}\end{array}$ & $\begin{array}{c}\mathrm{k}_{\mathrm{ex}} \times 10^{3} \\
/ \mathrm{s}-1\end{array}$ \\
\hline 2.2 & 5.45 & 1.63 & 4.11 & 1.74 & 2.52 \\
\hline 1.71 & 4.78 & 1.27 & 3.47 & 1.35 & 2.38 \\
\hline 1.28 & 4.04 & 0.951 & 2.89 & 1.01 & 2.05 \\
\hline 0.963 & 3.34 & 0.714 & 2.43 & 0.760 & 1.82 \\
\hline 0.642 & 2.59 & 0.476 & 1.88 & 0.507 & 1.47 \\
\hline 0.428 & 2.01 & 0.317 & 1.50 & 0.338 & 1.27 \\
\hline 0.285 & 1.58 & 0.211 & 1.19 & 0.225 & 1.05 \\
\hline \multicolumn{2}{|c|}{$\begin{aligned} \mathbf{k} & =2.0 \mathrm{M}^{-1} \mathrm{~s}^{-1} \\
\Delta \mathbf{k}^{a} & =0.3 \\
\mathbf{R}^{2} & =0.9841\end{aligned}$} & \multicolumn{2}{|c|}{$\begin{aligned} \mathbf{k} & =2.0 \mathrm{M}^{-1} \mathrm{~s}^{-1} \\
\Delta \mathbf{k}^{\mathbf{a}} & =0.13 \\
\mathbf{R}^{2} & =0.9943\end{aligned}$} & \multicolumn{2}{|c|}{$\begin{aligned} \mathbf{k} & =1.0 \mathrm{M}^{-1} \mathrm{~s}^{-1} \\
\Delta \mathbf{k}^{\mathbf{a}} & =0.16 \\
\mathbf{R}^{2} & =0.9633\end{aligned}$} \\
\hline
\end{tabular}

\begin{tabular}{cccc}
\hline \multicolumn{4}{c}{$1000 \mathrm{mM} \mathrm{CH}_{3} \mathrm{CO}_{2} \mathrm{H}$} \\
\hline $\begin{array}{c}{[\mathrm{DHZ}]} \\
/ \mathrm{mM}\end{array}$ & $\begin{array}{c}\mathrm{k}_{\mathrm{ex}} \times 10^{3} \\
/ \mathrm{s}-1\end{array}$ & $\begin{array}{c}{[\mathrm{DHZ}]} \\
/ \mathrm{mM}\end{array}$ & $\begin{array}{c}\mathrm{k}_{\mathrm{ex}} \times 10^{3} \\
/ \mathrm{s}-1\end{array}$ \\
\hline 1.11 & 1.91 & 1.17 & 1.76 \\
0.863 & 1.82 & 0.912 & 1.59 \\
0.648 & 1.69 & 0.684 & 1.44 \\
0.486 & 1.52 & 0.513 & 1.34 \\
0.364 & 1.38 & 0.385 & 1.23 \\
0.243 & 1.27 & 0.257 & 1.13
\end{tabular}

$\begin{array}{rlrl}\mathbf{k} & =0.76 \mathrm{M}^{-1} \mathrm{~s}^{-1} & \mathbf{k} & =0.68 \mathrm{M}^{-1} \mathrm{~s}^{-1} \\ \Delta \mathbf{k}^{\mathbf{a}} & =0.15 & \Delta \mathbf{k}^{\mathbf{a}} & =0.04 \\ \mathbf{R}^{2} & =0.9592 & \mathbf{R}^{2} & =0.9973\end{array}$

${ }^{a}$ calculated as the confidence interval of the slope for the $90 \%$ confidence level.

$k^{S}=1450 \pm 120 \mathrm{M}^{-1} \mathrm{~s}^{-1}$

$k_{5 \mathrm{mM} \mathrm{CH} 3 \mathrm{CO} 2 \mathrm{H}}^{S}=10.0 \pm 0.6 \mathrm{M}^{-1} \mathrm{~s}^{-1}$

$k^{S}{ }_{10 \mathrm{mM} \mathrm{CH}} \mathrm{CO}_{2 \mathrm{H}}=6.1 \pm 0.4 \mathrm{M}^{-1} \mathrm{~s}^{-1}$

$k_{50 \mathrm{mM} \mathrm{CH}}^{S \mathrm{CO}_{2 H}} \quad=2.0 \pm 0.2 \mathrm{M}^{-1} \mathrm{~s}^{-1}$

$k_{100 \mathrm{mM} \mathrm{CH} 3 \mathrm{CO} 2}{ }^{5}=1.0 \pm 0.2 \mathrm{M}^{-1} \mathrm{~s}^{-1}$

$k^{s}{ }_{1000 \mathrm{mM} \mathrm{CH}} \mathrm{CO}_{2 \mathrm{H}}=0.72 \pm 0.1 \mathrm{M}^{-1} \mathrm{~s}^{-1}$ 
Table S7. Kinetic data for the reaction of dpph $^{\bullet}$ with IE in methanol. Symbols are the same as described in Table S1.

\begin{tabular}{|c|c|c|c|c|c|c|c|}
\hline $\begin{array}{c}{[\mathrm{IE}]} \\
/ \mathrm{mM}\end{array}$ & $\begin{array}{l}\mathrm{k}_{\mathrm{ex}} \\
/ \mathrm{s}-1\end{array}$ & $\begin{array}{l}{[\mathrm{IE}]} \\
/ \mathrm{mM}\end{array}$ & $\begin{array}{l}k_{e x} \\
/ s-1 \\
\end{array}$ & $\begin{array}{c}{[\mathrm{IE}]} \\
/ \mathrm{mM} \\
\end{array}$ & $\begin{array}{l}k_{e x} \\
/ s-1\end{array}$ & $\begin{array}{c}{[\mathrm{IE}]} \\
/ \mathrm{mM}\end{array}$ & $\begin{array}{l}k_{e x} \\
/ s-1\end{array}$ \\
\hline 6.17 & 7.20 & 1.28 & 1.25 & 2.94 & 2.42 & 1.26 & 1.29 \\
\hline 2.94 & 2.42 & 1.00 & 1.05 & 1.76 & 1.79 & 0.978 & 1.04 \\
\hline 1.76 & 1.79 & 0.749 & 0.810 & 0.635 & 0.492 & 0.734 & 0.844 \\
\hline 1.06 & 0.548 & 0.535 & 0.589 & 0.381 & 0.350 & 0.550 & 0.677 \\
\hline 0.635 & 0.492 & 0.357 & 0.400 & 0.228 & 0.121 & 0.413 & 0.527 \\
\hline 0.381 & 0.350 & 0.214 & 0.198 & 0.137 & 0.078 & 0.309 & 0.396 \\
\hline 0.228 & 0.121 & 0.128 & 0.135 & 0.082 & 0.041 & 0.232 & 0.308 \\
\hline 0.137 & 0.078 & 0.077 & 0.097 & 0.049 & 0.049 & 0.174 & 0.249 \\
\hline 0.082 & 0.041 & & & & & 0.087 & 0.132 \\
\hline 0.049 & 0.049 & & & & & & \\
\hline \multicolumn{2}{|c|}{$\begin{aligned} \mathbf{k} & =1140 \mathrm{M}^{-1} \mathrm{~s}^{-1} \\
\Delta \mathbf{k}^{\mathbf{a}} & =110 \\
\mathbf{R}^{2} & =0.9784\end{aligned}$} & \multicolumn{2}{|c|}{$\begin{aligned} \mathbf{k} & =994 \mathrm{M}^{-1} \mathrm{~s}^{-1} \\
\Delta \mathbf{k}^{\mathbf{a}} & =62 \\
\mathbf{R}^{2} & =0.9918\end{aligned}$} & \multicolumn{2}{|c|}{$\begin{aligned} \mathbf{k} & =877 \mathrm{M}^{-1} \mathrm{~s}^{-1} \\
\Delta \mathbf{k}^{\mathbf{a}} & =86 \\
\mathbf{R}^{2} & =0.9822\end{aligned}$} & \multicolumn{2}{|c|}{$\begin{aligned} \mathbf{k} & =979 \mathrm{M}^{-1} \mathrm{~s}^{-1} \\
\Delta \mathbf{k}^{\mathbf{a}} & =59 \\
\mathbf{R}^{2} & =0.9932\end{aligned}$} \\
\hline
\end{tabular}

\begin{tabular}{cc}
\hline$[\mathrm{IE}]$ & $\mathrm{k}_{\mathrm{ex}}$ \\
$/ \mathrm{mM}$ & $/ \mathrm{s}-1$ \\
\hline 1.745 & 2.14 \\
1.357 & 1.74 \\
1.018 & 1.43 \\
0.727 & 1.18 \\
0.485 & 0.862 \\
0.291 & 0.546
\end{tabular}

$$
\begin{aligned}
\mathbf{k} & =1054 \mathrm{M}^{-1} \mathrm{~s}^{-1} \\
\Delta \mathbf{k}^{\mathbf{a}} & =105 \\
\mathbf{R}^{2} & =0.9897
\end{aligned}
$$

${ }^{a}$ calculated as the confidence interval of the slope for the $90 \%$ confidence level. 
Table S8. Kinetic data for the reaction of $\mathrm{dpph}^{\bullet}$ with IE in acidified methanol. Symbols are the same as described in Table S1.

\begin{tabular}{|c|c|c|c|c|c|c|c|}
\hline \multicolumn{4}{|c|}{$5 \mathrm{mM} \mathrm{CH}_{3} \mathrm{CO}_{2} \mathrm{H}$} & \multicolumn{4}{|c|}{$10 \mathrm{mM} \mathrm{CH}_{3} \mathrm{CO}_{2} \mathrm{H}$} \\
\hline $\begin{array}{l}{[\mathrm{IE}]} \\
/ \mathrm{mM}\end{array}$ & $\begin{array}{c}\mathrm{k}_{\mathrm{ex}} \times 10^{3} \\
/ \mathrm{s}-1\end{array}$ & $\begin{array}{l}{[\mathrm{IE}]} \\
/ \mathrm{mM}\end{array}$ & $\begin{array}{c}\mathrm{k}_{\mathrm{ex}} \times 10^{3} \\
/ \mathrm{s}-1\end{array}$ & $\begin{array}{l}{[\mathrm{IE}]} \\
/ \mathrm{mM}\end{array}$ & $\begin{array}{c}\mathrm{k}_{\mathrm{ex}} \times 10^{3} \\
/ \mathrm{s}-1\end{array}$ & $\begin{array}{l}\text { [IE] } \\
/ \mathrm{mM}\end{array}$ & $\begin{array}{c}\mathrm{k}_{\mathrm{ex}} \times 10^{3} \\
/ \mathrm{s}-1\end{array}$ \\
\hline 5.47 & 80.43 & 3.42 & 36.39 & 4.89 & 38.5 & 7.03 & 57.44 \\
\hline 4.10 & 62.67 & 2.56 & 30.15 & 3.80 & 32.0 & 5.47 & 48.77 \\
\hline 2.93 & 46.46 & 1.83 & 24.18 & 2.85 & 25.7 & 4.10 & 39.87 \\
\hline 1.95 & 33.30 & 1.22 & 18.08 & 2.04 & 19.8 & 2.93 & 30.67 \\
\hline 1.17 & 22.79 & 0.733 & 12.48 & 1.36 & 14.3 & 1.95 & 22.53 \\
\hline 0.703 & 15.13 & 0.440 & 8.84 & 0.815 & 10.0 & 1.17 & 15.15 \\
\hline \multicolumn{2}{|c|}{$\begin{aligned} \mathbf{k} & =14 \mathrm{M}^{-1} \mathrm{~s}^{-1} \\
\Delta \mathbf{k}^{\mathbf{a}} & =0.28 \\
\mathbf{R}^{2} & =0.9996\end{aligned}$} & \multicolumn{2}{|c|}{$\begin{aligned} \mathbf{k} & =9.3 \mathrm{M}^{-1} \mathrm{~s}^{-1} \\
\Delta \mathbf{k}^{\mathbf{a}} & =0.9 \\
\mathbf{R}^{2} & =0.9902\end{aligned}$} & \multicolumn{2}{|c|}{$\begin{aligned} \mathbf{k} & =7.2 \mathrm{M}^{-1} \mathrm{~s}^{-1} \\
\Delta \mathbf{k}^{\mathbf{a}} & =0.37 \\
\mathbf{R}^{2} & =0.9964\end{aligned}$} & \multicolumn{2}{|c|}{$\begin{aligned} \mathbf{k} & =7.5 \mathrm{M}^{-1} \mathrm{~s}^{-1} \\
\Delta \mathbf{k}^{a} & =0.61 \\
\mathbf{R}^{2} & =0.9906\end{aligned}$} \\
\hline \multicolumn{4}{|c|}{$50 \mathrm{mM} \mathrm{CH}_{3} \mathrm{CO}_{2} \mathrm{H}$} & \multicolumn{4}{|c|}{$100 \mathrm{mM} \mathrm{CH}_{3} \mathrm{CO}_{2} \mathrm{H}$} \\
\hline $\begin{array}{l}{[\mathrm{IE}]} \\
/ \mathrm{mM}\end{array}$ & $\begin{array}{c}\mathrm{k}_{\mathrm{ex}} \times 10^{3} \\
/ \mathrm{s}-1\end{array}$ & $\begin{array}{l}{[\mathrm{IE}]} \\
/ \mathrm{mM}\end{array}$ & $\begin{array}{c}\mathrm{k}_{\mathrm{ex}} \times 10^{3} \\
/ \mathrm{s}-1\end{array}$ & $\begin{array}{l}{[\mathrm{IE}]} \\
/ \mathrm{mM}\end{array}$ & $\begin{array}{c}\mathrm{k}_{\mathrm{ex}} \times 10^{3} \\
/ \mathrm{s}-1\end{array}$ & $\begin{array}{l}\text { [IE] } \\
/ \mathrm{mM}\end{array}$ & $\begin{array}{c}\mathrm{k}_{\mathrm{ex}} \times 10^{3} \\
/ \mathrm{s}-1\end{array}$ \\
\hline 4.89 & 30.2 & 7.03 & 42.3 & 4.89 & 32.8 & 7.03 & 40.4 \\
\hline 3.80 & 23.4 & 5.47 & 34.0 & 3.80 & 27.4 & 5.47 & 33.0 \\
\hline 2.85 & 21.0 & 4.10 & 27.2 & 2.85 & 24.1 & 4.10 & 26.0 \\
\hline 2.04 & 16.5 & 2.93 & 21.0 & 2.04 & 18.9 & 2.93 & 20.2 \\
\hline 1.36 & 12.2 & 1.95 & 14.5 & 1.36 & 13.6 & 1.95 & 14.6 \\
\hline 0.815 & 8.66 & 1.17 & 9.77 & 0.815 & 9.19 & 1.17 & 9.84 \\
\hline 0.489 & 6.02 & 0.703 & 6.71 & 0.489 & 6.33 & 0.703 & 6.64 \\
\hline \multicolumn{2}{|c|}{$\begin{aligned} \mathbf{k} & =5.3 \quad \mathrm{M}^{-1} \mathrm{~s}^{-1} \\
\Delta \mathrm{k}^{a} & =0.6 \\
\mathbf{R}^{2} & =0.9849\end{aligned}$} & \multicolumn{2}{|c|}{$\begin{aligned} \mathbf{k} & =5.6 \mathrm{M}^{-1} \mathrm{~s}^{-1} \\
\Delta \mathrm{k}^{a} & =0.3 \\
\mathbf{R}^{2} & =0.9975\end{aligned}$} & \multicolumn{2}{|c|}{$\begin{aligned} \mathbf{k} & =6.0 \mathrm{M}^{-1} \mathrm{~s}^{-1} \\
\Delta \mathrm{k}^{a} & =0.75 \\
\mathbf{R}^{2} & =0.9783\end{aligned}$} & \multicolumn{2}{|c|}{$\begin{aligned} \mathbf{k} & =5.3 \quad \mathrm{M}^{-1} \mathrm{~s}^{-1} \\
\Delta \mathrm{k}^{a} & =0.3 \\
\mathbf{R}^{2} & =0.9974\end{aligned}$} \\
\hline
\end{tabular}


Table S8. continued

\begin{tabular}{|c|c|c|c|c|c|}
\hline \multicolumn{2}{|c|}{$100 \mathrm{mM} \mathrm{CH}_{3} \mathrm{CO}_{2} \mathrm{H}$} & \multicolumn{4}{|c|}{$1000 \mathrm{mM} \mathrm{CH}_{3} \mathrm{CO}_{2} \mathrm{H}$} \\
\hline $\begin{array}{l}\text { [IE] } \\
/ \mathrm{mM}\end{array}$ & $\begin{array}{c}\mathrm{k}_{\mathrm{ex}} \times 10^{3} \\
/ \mathrm{s}-1\end{array}$ & $\begin{array}{l}\text { [IE] } \\
/ \mathrm{mM}\end{array}$ & $\begin{array}{c}\mathrm{k}_{\mathrm{ex}} \times 10^{3} \\
/ \mathrm{s}-1\end{array}$ & $\begin{array}{l}\text { [IE] } \\
/ \mathrm{mM}\end{array}$ & $\begin{array}{c}\mathrm{k}_{\mathrm{ex}} \times 10^{3} \\
/ \mathrm{s}-1\end{array}$ \\
\hline 2.09 & 9.40 & 4.58 & 31.8 & 6.11 & 43.1 \\
\hline 1.39 & 6.93 & 3.57 & 26.0 & 4.75 & 36.6 \\
\hline 0.929 & 4.99 & 2.67 & 20.9 & 3.57 & 29.4 \\
\hline 0.619 & 3.63 & 1.91 & 16.1 & 2.55 & 22.3 \\
\hline 0.413 & 2.83 & 1.27 & 11.8 & 1.70 & 16.0 \\
\hline 0.275 & 2.20 & 0.764 & 8.16 & 1.02 & 11.1 \\
\hline 0.183 & 1.74 & 0.458 & 5.73 & 0.611 & 7.60 \\
\hline \multicolumn{2}{|c|}{$\begin{aligned} \mathbf{k} & =4.8 \mathrm{M}^{-1} \mathrm{~s}^{-1} \\
\Delta \mathbf{k}^{\mathbf{a}} & =0.2 \\
\mathbf{R}^{2} & =0.9981\end{aligned}$} & \multicolumn{2}{|c|}{$\begin{aligned} \mathbf{k} & =6.3 \quad \mathrm{M}^{-1} \mathrm{~s}^{-1} \\
\Delta \mathbf{k}^{\mathbf{a}} & =0.3 \\
\mathbf{R}^{2} & =0.9973\end{aligned}$} & \multicolumn{2}{|c|}{$\begin{aligned} \mathbf{k} & =6.5 \mathrm{M}^{-1} \mathrm{~s}^{-1} \\
\Delta \mathbf{k}^{\mathbf{a}} & =0.5 \\
\mathbf{R}^{\mathbf{2}} & =0.9930\end{aligned}$} \\
\hline
\end{tabular}

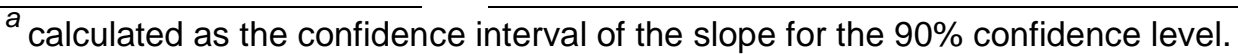

$k^{S}=(1.0 \pm 0.2) \times 10^{3} \quad M^{-1} s^{-1}$

$k_{5 \mathrm{mM} \mathrm{CH}} \mathrm{CH}^{\mathrm{COH}}=12 \pm 2 \mathrm{M}^{-1} \mathrm{~s}^{-1}$

$k^{s}{ }_{10 \mathrm{mM} \mathrm{CH}} \mathrm{CHO}_{2 \mathrm{H}}=7.4 \pm 0.5 \mathrm{M}^{-1} \mathrm{~s}^{-1}$

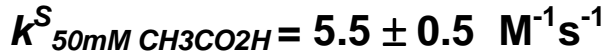

$k^{S}{ }_{100 \mathrm{mM} \mathrm{CH}} \mathrm{CHO}_{\mathrm{CH}}=5.4 \pm 0.5 \mathrm{M}^{-1} \mathrm{~s}^{-1}$

$k^{S}{ }_{1000 \mathrm{mM} \mathrm{CH} 3 \mathrm{CO} 2 \mathrm{H}}=6.4 \pm 0.5 \mathrm{M}^{-1} \mathrm{~s}^{-1}$

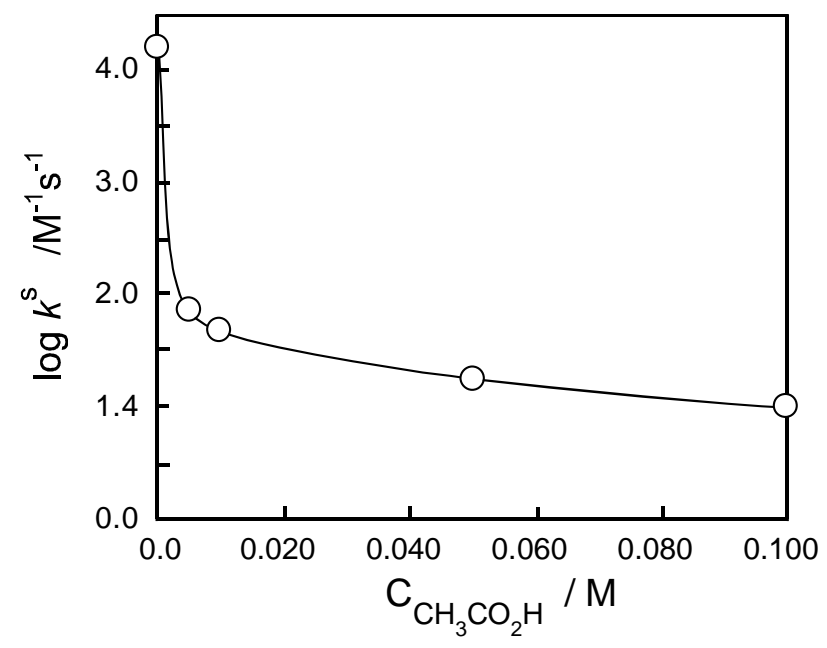

Figure S1. Dependence of the $\log k^{s}$ vs. concentration of acetic acid in methanol. The error bars are within the experimental points. 
Table S9. Kinetic data for the reaction of $\mathbf{d p p h}^{\bullet}$ with acetylacetone (AcAc) in methanol. Symbols are the same as described in Table S1.

\begin{tabular}{|c|c|c|c|}
\hline $\begin{array}{c}{[\mathrm{AcAc}]} \\
/ \mathrm{mM}\end{array}$ & $\begin{array}{c}\mathrm{k}_{\mathrm{ex}} \times 10^{3} \\
/ \mathrm{s}-1\end{array}$ & $\begin{array}{c}{[\mathrm{AcAc}]} \\
/ \mathrm{mM}\end{array}$ & $\begin{array}{c}\mathrm{k}_{\mathrm{ex}} \times 10^{3} \\
/ \mathrm{s}-1\end{array}$ \\
\hline 34.4 & 1.14 & 10.1 & 0.534 \\
\hline 26.8 & 0.957 & 7.87 & 0.463 \\
\hline 20.8 & 0.788 & 6.12 & 0.365 \\
\hline 16.2 & 0.637 & 4.76 & 0.293 \\
\hline 12.6 & 0.508 & 3.70 & 0.251 \\
\hline 9.80 & 0.414 & 2.88 & 0.188 \\
\hline \multirow[t]{3}{*}{7.62} & 0.340 & 2.24 & 0.149 \\
\hline & & 1.74 & 0.139 \\
\hline & & 1.35 & 0.110 \\
\hline \multicolumn{2}{|c|}{$\mathbf{k}=0.030 \mathrm{M}^{-1} \mathrm{~s}^{-1}$} & \multicolumn{2}{|c|}{$\mathbf{k}=0.050 \mathrm{M}^{-1} \mathrm{~s}^{-1}$} \\
\hline \multicolumn{2}{|c|}{$\Delta \mathbf{k}^{a}=0.002$} & \multicolumn{2}{|c|}{$\Delta \mathbf{k}^{a}=0.003$} \\
\hline
\end{tabular}

a calculated as the confidence interval of the slope for the $90 \%$ confidence level.

$k^{S}=0.04 \pm 0.01 \mathrm{M}^{-1} \mathrm{~s}^{-1}$

Figure S2. Plot of $k_{e x}$ vs. concentration of [AcAc] in methanol. Chart a: Based on the AcAc concentration range (7.6-200 mM). The $k_{e x}$ values at $[A c A c]>34.4 \mathrm{mM}$ have not been included in Table S9. The bimolecular rate rate constant, $\boldsymbol{k}^{\boldsymbol{s}}$, was calculated for straight line portion of the $k_{e x}$ vs. [AcAc] plot, Chart b.
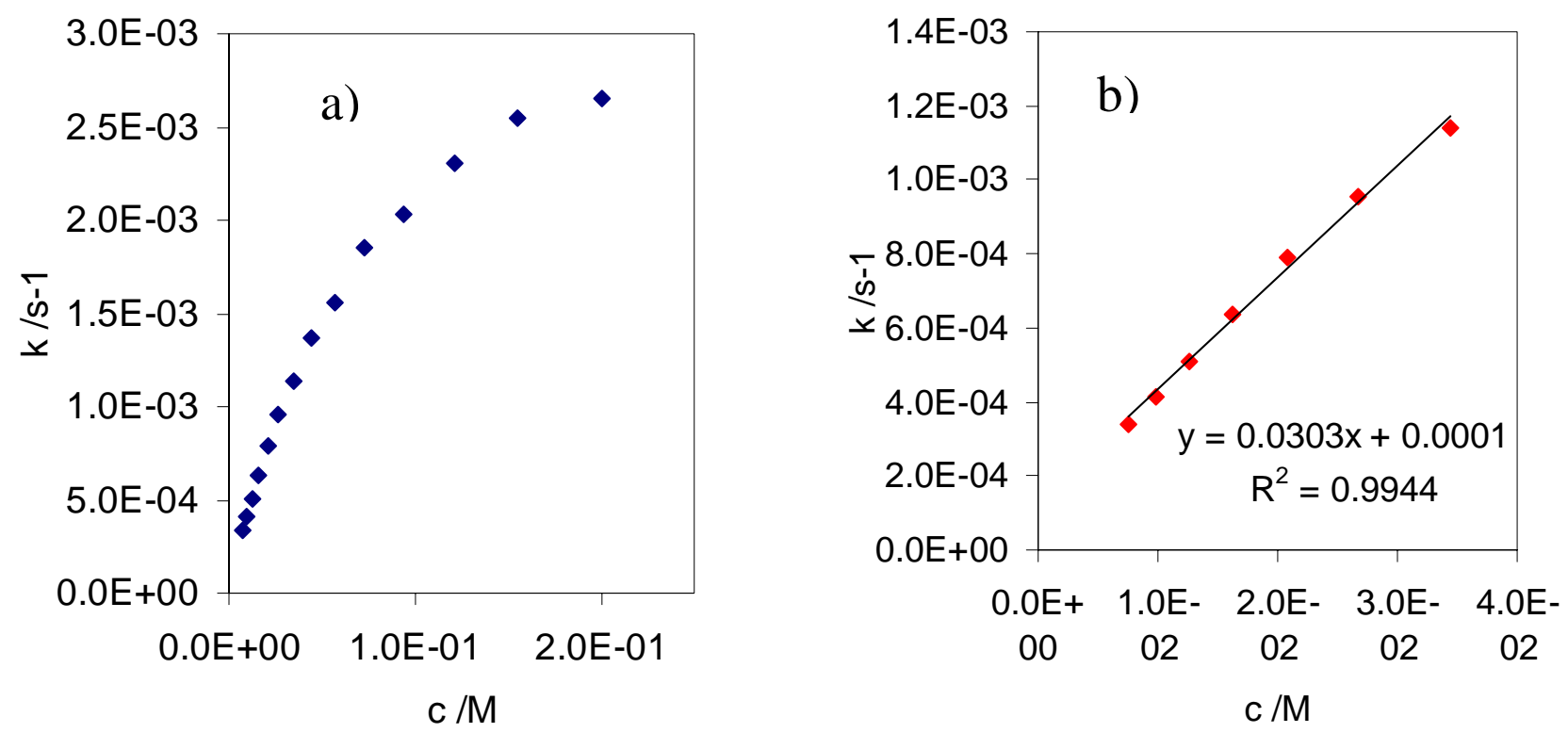
Table S10. Kinetic data for the reaction of $\mathrm{dpph}^{\bullet}$ with $\mathrm{CU}$ in ethanol. Symbols are the same as described in Table S1.

\begin{tabular}{|c|c|c|c|c|c|c|c|}
\hline \multicolumn{2}{|c|}{ neat $\mathrm{EtOH}$} & \multicolumn{2}{|c|}{ neat EtOH } & \multicolumn{2}{|c|}{$5 \mathrm{mM} \mathrm{CH}_{3} \mathrm{CO}_{2} \mathrm{H}$} & \multicolumn{2}{|c|}{$10 \mathrm{mM} \mathrm{CH}_{3} \mathrm{CO}_{2} \mathrm{H}$} \\
\hline $\begin{array}{c}{[\mathrm{CU}] \times 10^{5}} \\
/ \mathrm{M}\end{array}$ & $\begin{array}{l}\mathrm{k}_{\mathrm{ex}} \\
/ \mathrm{s}-1\end{array}$ & $\begin{array}{c}{[\mathrm{CU}] \times 10^{5}} \\
/ \mathrm{M}\end{array}$ & $\begin{array}{l}\mathrm{k}_{\mathrm{ex}} \\
/ \mathrm{s}-1\end{array}$ & $\begin{array}{c}{[\mathrm{CU}] \times 10^{5} \mathrm{~K}} \\
/ \mathrm{M}\end{array}$ & $\begin{array}{c}\mathrm{k}_{\mathrm{ex}} \times 10^{3} \\
/ \mathrm{s}-1\end{array}$ & $\begin{array}{c}{[\mathrm{CU}] \times 10^{5}} \\
/ \mathrm{M}\end{array}$ & $\begin{array}{c}\mathrm{k}_{\mathrm{ex}} \times 10^{3} \\
/ \mathrm{s}-1\end{array}$ \\
\hline 22.9 & 1.98 & 37.84 & 4.10 & 32.2 & 19.3 & 37.8 & 17.7 \\
\hline 17.8 & 1.73 & 29.43 & 3.63 & 23.0 & 16.0 & 29.4 & 16.3 \\
\hline 13.8 & 1.20 & 17.80 & 2.17 & 15.3 & 11.9 & 22.9 & 13.2 \\
\hline 10.8 & 1.18 & 13.85 & 1.92 & 9.2 & 7.79 & 17.8 & 10.0 \\
\hline 8.4 & 0.866 & 10.77 & 1.72 & 5.5 & 4.98 & 13.8 & 7.55 \\
\hline 6.5 & 0.552 & 8.377 & 1.36 & 3.3 & 2.87 & 10.8 & 5.99 \\
\hline 3.9 & 0.483 & 6.515 & 0.739 & 2.0 & 1.83 & 8.38 & 5.45 \\
\hline 3.1 & 0.436 & 5.067 & 0.525 & & & 6.52 & 4.36 \\
\hline 2.4 & 0.316 & 3.941 & 0.469 & & & 5.07 & 3.13 \\
\hline & & & & & & 3.94 & 2.95 \\
\hline & & & & & & 3.07 & 2.20 \\
\hline $\begin{aligned} \mathbf{k} & =\varepsilon \\
\Delta \mathbf{k}^{a} & =c \\
\mathbf{R}^{2} & =c\end{aligned}$ & $\begin{array}{l}<10^{3} \mathrm{M}^{-1} \mathrm{~s}^{-1} \\
10^{3} \\
768\end{array}$ & $\begin{aligned} \mathbf{k} & =1 \\
\Delta \mathbf{k}^{\mathbf{a}} & =1 \\
\mathbf{R}^{2} & =0\end{aligned}$ & $\begin{array}{l}10^{3} \mathrm{M}^{-1} \mathrm{~s}^{-1} \\
10^{3} \\
28\end{array}$ & $\begin{aligned} \mathbf{k} & =5 \\
\Delta \mathbf{k}^{\mathbf{a}} & =7 \\
\mathbf{R}^{2} & =0\end{aligned}$ & $\begin{array}{l}9 \mathrm{M}^{-1} \mathrm{~s}^{-1} \\
.5 \\
.9781\end{array}$ & $\begin{aligned} \mathbf{k} & = \\
\Delta \mathbf{k}^{\mathbf{a}} & = \\
\mathbf{R}^{2} & =\end{aligned}$ & $\begin{array}{l}8.0 \mathrm{M}^{-1} \mathrm{~s}^{-1} \\
0 \\
9827\end{array}$ \\
\hline
\end{tabular}

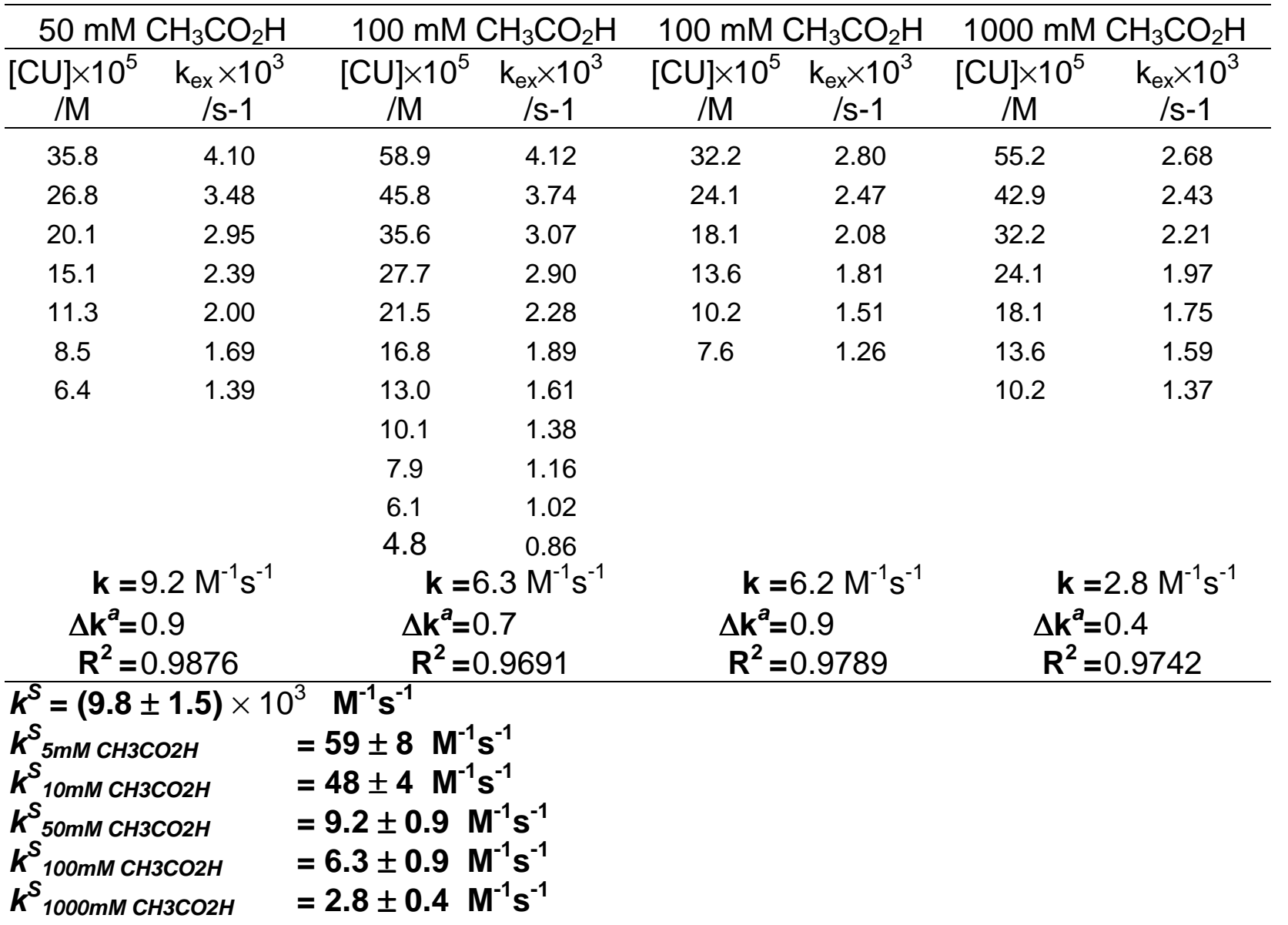


Table S11. Kinetic data for the reaction of $\mathbf{d p p h}^{\bullet}$ with $\mathrm{DHZ}$ in ethanol. Symbols are the same as described in Table $\mathbf{S 1 .}$

\begin{tabular}{|c|c|c|c|c|c|}
\hline \multicolumn{2}{|c|}{ neat EtOH } & \multicolumn{2}{|c|}{ neat EtOH } & \multicolumn{2}{|c|}{ neat EtOH } \\
\hline $\begin{array}{l}{[\mathrm{DHZ}]} \\
/ \mathrm{mM}\end{array}$ & $\begin{array}{c}\mathrm{k}_{\mathrm{ex}} \\
\mathrm{/s}-1\end{array}$ & $\begin{array}{c}{[\mathrm{DHZ}]} \\
/ \mathrm{mM}\end{array}$ & $\begin{array}{l}\mathrm{k}_{\mathrm{ex}} \\
/ \mathrm{s}-1\end{array}$ & $\begin{array}{c}{[\mathrm{DHZ}]} \\
/ \mathrm{mM}\end{array}$ & $\begin{array}{c}\mathrm{k}_{\mathrm{ex}} \\
\mathrm{s}-1\end{array}$ \\
\hline 1.57 & 1.18 & 0.966 & 0.807 & 2.24 & 2.62 \\
\hline 1.22 & 1.01 & 0.724 & 0.680 & 1.74 & 2.12 \\
\hline 0.949 & 0.807 & 0.543 & 0.589 & 1.35 & 1.90 \\
\hline 0.738 & 0.680 & 0.407 & 0.416 & 1.05 & 1.81 \\
\hline 0.574 & 0.589 & 0.306 & 0.325 & 0.818 & 1.70 \\
\hline 0.446 & 0.416 & 0.229 & 0.270 & 0.636 & 1.25 \\
\hline 0.347 & 0.325 & 0.172 & 0.198 & 0.495 & 1.14 \\
\hline 0.270 & 0.270 & & & 0.385 & 1.01 \\
\hline 0.193 & 0.198 & & & 0.192 & 0.795 \\
\hline \multicolumn{2}{|c|}{$\begin{aligned} \mathbf{k} & =725 \mathrm{M}^{-1} \mathrm{~s}^{-1} \\
\Delta \mathbf{k}^{\mathbf{a}} & =65 \\
\mathbf{R}^{2} & =0.9834\end{aligned}$} & \multicolumn{2}{|c|}{$\begin{aligned} \mathbf{k} & =782 \mathrm{M}^{-1} \mathrm{~s}^{-1} \\
\Delta \mathbf{k}^{\mathbf{a}} & =108 \\
\mathbf{R}^{2} & =0.9737\end{aligned}$} & \multicolumn{2}{|c|}{$\begin{aligned} \mathbf{k} & =852 \mathrm{M}^{-1} \mathrm{~s}^{-1} \\
\Delta \mathbf{k}^{\mathbf{a}} & =128 \\
\mathbf{R}^{2} & =0.9552\end{aligned}$} \\
\hline
\end{tabular}

${ }^{a}$ calculated as the confidence interval of the slope for the $90 \%$ confidence level.

$k^{S}=(790 \pm 70) \quad M^{-1} s^{-1}$ 
Table S12. Kinetic data for the reaction of dpph ${ }^{\bullet}$ with $\mathrm{DHZ}$ in acidified ethanol. Symbols are the same as described in Table S1.

\begin{tabular}{|c|c|c|c|c|c|c|c|}
\hline \multicolumn{4}{|c|}{$5 \mathrm{mM} \mathrm{CH}_{3} \mathrm{CO}_{2} \mathrm{H}$} & \multicolumn{2}{|c|}{$10 \mathrm{mM} \mathrm{CH}_{3} \mathrm{CO}_{2} \mathrm{H}$} & \multicolumn{2}{|c|}{$50 \mathrm{mM} \mathrm{CH}_{3} \mathrm{CO}_{2} \mathrm{H}$} \\
\hline $\begin{array}{c}{[\mathrm{DHZ}]} \\
/ \mathrm{mM}\end{array}$ & $\begin{array}{c}\mathrm{k}_{\mathrm{ex}} \times 10^{3} \\
/ \mathrm{s}-1\end{array}$ & $\begin{array}{c}{[\mathrm{DHZ}]} \\
/ \mathrm{mM}\end{array}$ & $\begin{array}{c}\mathrm{k}_{\mathrm{ex}} \times 10^{3} \\
/ \mathrm{s}-1\end{array}$ & $\begin{array}{c}\text { [DHZ] } \\
/ \mathrm{mM}\end{array}$ & $\begin{array}{c}\mathrm{kex} \times 10^{3} \\
/ \mathrm{s}-1\end{array}$ & $\begin{array}{c}\text { [DHZ] } \\
/ \mathrm{mM}\end{array}$ & $\begin{array}{l}\mathrm{k}_{\mathrm{ex}} \times 10^{3} \\
/ \mathrm{s}-1\end{array}$ \\
\hline 1.95 & 21.4 & 1.78 & 17.2 & 1.69 & 13.4 & 1.45 & 4.33 \\
\hline 1.51 & 17.8 & 1.38 & 14.2 & 1.31 & 11.2 & 1.13 & 3.47 \\
\hline 1.14 & 14.2 & 1.04 & 11.6 & 0.983 & 8.94 & 0.844 & 2.86 \\
\hline 0.851 & 11.2 & 0.778 & 8.81 & 0.738 & 7.15 & 0.633 & 2.32 \\
\hline 0.568 & 8.14 & 0.519 & 6.40 & 0.492 & 5.22 & 0.422 & 1.78 \\
\hline \multirow[t]{2}{*}{0.378} & 5.81 & 0.346 & 4.74 & 0.328 & 3.79 & 0.281 & 1.32 \\
\hline & & 0.230 & 3.98 & 0.219 & 2.68 & 0.188 & 0.987 \\
\hline \multicolumn{2}{|c|}{$\begin{aligned} \mathbf{k} & =10 \mathrm{M}^{-1} \mathrm{~s}^{-1} \\
\Delta \mathbf{k}^{\mathbf{a}} & =0.6 \\
\mathbf{R}^{2} & =0.9962\end{aligned}$} & \multicolumn{2}{|c|}{$\begin{aligned} \mathbf{k} & =8.8 \mathrm{M}^{-1} \mathrm{~s}^{-1} \\
\Delta \mathbf{k}^{\mathbf{a}} & =0.4 \\
\mathbf{R}^{2} & =0.9969\end{aligned}$} & \multicolumn{2}{|c|}{$\begin{aligned} \mathbf{k} & =7.3 \mathrm{M}^{-1} \mathrm{~s}^{-1} \\
\Delta \mathbf{k}^{\mathbf{a}} & =0.5 \\
\mathbf{R}^{2} & =0.9948\end{aligned}$} & \multicolumn{2}{|c|}{$\begin{aligned} \mathbf{k} & =2.6 \mathrm{M}^{-1} \mathrm{~s}^{-1} \\
\Delta \mathbf{k}^{\mathbf{a}} & =0.13 \\
\mathbf{R}^{2} & =0.9964\end{aligned}$} \\
\hline \multicolumn{2}{|c|}{$100 \mathrm{mM} \mathrm{CH}_{3} \mathrm{CO}_{2} \mathrm{H}$} & \multicolumn{3}{|c|}{$1000 \mathrm{mM} \mathrm{CH}_{3} \mathrm{CO}_{2} \mathrm{H}$} & & & \\
\hline $\begin{array}{l}\mathrm{DHZ}] \\
/ \mathrm{mM}\end{array}$ & $\begin{array}{c}\mathrm{k}_{\mathrm{ex}} \times 10^{3} \\
/ \mathrm{s}-1\end{array}$ & \multicolumn{2}{|c|}{$\begin{array}{c}{[\mathrm{DHZ}]} \\
/ \mathrm{mM}\end{array}$} & $\begin{array}{c}\mathrm{k}_{\mathrm{ex}} \times 10^{3} \\
/ \mathrm{s}-1\end{array}$ & & & \\
\hline 1.45 & 3.11 & \multicolumn{2}{|c|}{1.57} & 2.48 & & & \\
\hline 1.13 & 2.68 & \multicolumn{2}{|c|}{1.22} & 2.13 & & & \\
\hline 0.844 & 2.21 & \multicolumn{2}{|c|}{0.915} & 1.82 & & & \\
\hline 0.633 & 1.79 & \multicolumn{2}{|c|}{0.686} & 1.57 & & & \\
\hline 0.422 & 1.39 & \multicolumn{2}{|c|}{0.458} & 1.27 & & & \\
\hline 0.281 & 1.09 & 0.3 & & 1.01 & & & \\
\hline 0.188 & 0.840 & 0.2 & 03 & 0.840 & & & \\
\hline \multicolumn{2}{|c|}{$\begin{aligned} \mathbf{k} & =1.8 \mathrm{M}^{-1} \mathbf{s}^{-1} \\
\Delta \mathbf{k}^{\mathbf{a}} & =0.14 \\
\mathbf{R}^{2} & =0.9922\end{aligned}$} & & $\begin{array}{l}k=1.2 \mathrm{M} \\
\mathrm{k}^{\mathrm{a}}=0.1 \\
2^{2}=0.989\end{array}$ & $\begin{array}{l}1^{-1} s^{-1} \\
2 \\
\end{array}$ & & & \\
\hline calculat & as the confid & ce interva & al of the slc & ope for the 90 & onfidenc & & \\
\hline 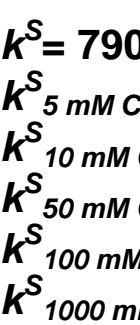 & $\begin{array}{l}70 \mathrm{M}^{-1} \mathrm{~s}^{-1} \\
\mathrm{CO} 2 \mathrm{H} \\
3 \mathrm{CO} 2 \mathrm{H} \\
3 \mathrm{CO} 2 \mathrm{H} \\
\mathrm{H} 3 \mathrm{CO} 2 \mathrm{H}= \\
\mathrm{H} 3 \mathrm{CO}_{2} \mathrm{H}=\end{array}$ & $\begin{array}{l}9.4 \pm 0.6 \\
7.3 \pm 0.5 \\
2.6 \pm 0.2 \\
1.8 \pm 0.1 \\
1.2 \pm 0.1\end{array}$ & $\begin{array}{l}M^{-1} s^{-1} \\
M^{-1} s^{-1} \\
M^{-1} s^{-1} \\
M^{-1} s^{-1} \\
M^{-1} s^{-1}\end{array}$ & & & & \\
\hline
\end{tabular}


Table S13. Kinetic data for the reaction of $\mathrm{dpph}^{\bullet}$ with IE in pure ethanol and acidified ethanol. Symbols are the same as described in Table S1.

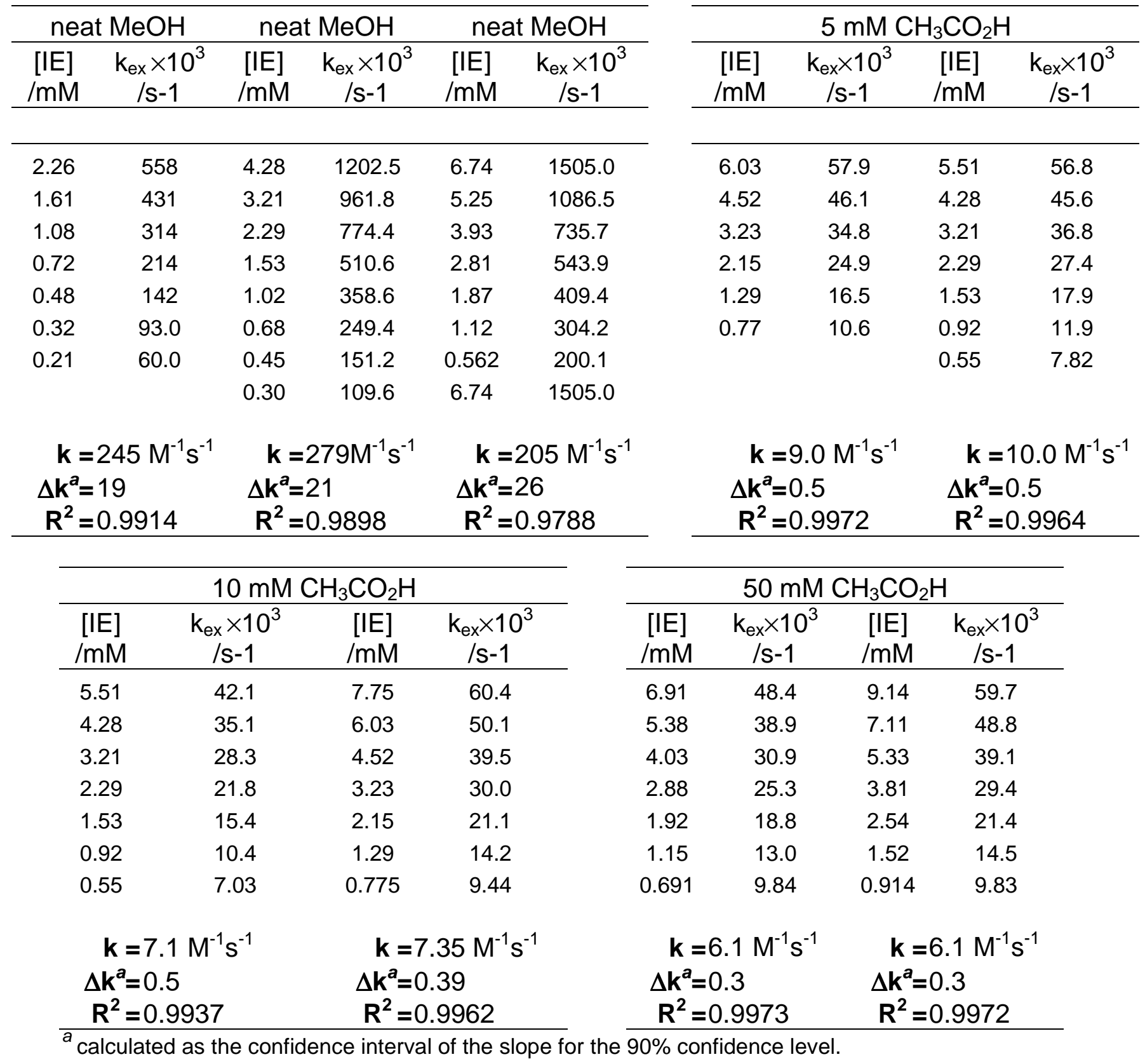


Table S15. Kinetic data for the reaction of $\mathrm{dpph}^{\bullet}$ with $\mathrm{CU}$ in ethyl acetate and in ethyl acetate containing acetic acid. Symbols are the same as described in Table S1.

\begin{tabular}{|c|c|c|c|}
\hline \multicolumn{2}{|c|}{ ethyl acetate } & \multicolumn{2}{|c|}{ ethyl acetate } \\
\hline $\begin{array}{l}{[\mathrm{CU}]} \\
/ \mathrm{mM} \\
\end{array}$ & $\begin{array}{c}\mathrm{k}_{\mathrm{ex}} \times 10^{3} \\
/ \mathrm{s}-1\end{array}$ & $\begin{array}{l}{[\mathrm{CU}]} \\
/ \mathrm{mM}\end{array}$ & $\begin{array}{c}\mathrm{k}_{\mathrm{ex}} \times 10^{3} \\
/ \mathrm{s}-1\end{array}$ \\
\hline 1.42 & 13.2 & 1.77 & 15.2 \\
\hline 0.95 & 7.69 & 1.33 & 11.4 \\
\hline 0.64 & 4.79 & 1.00 & 8.16 \\
\hline 0.42 & 3.75 & 0.75 & 6.11 \\
\hline 0.33 & 2.80 & 0.56 & 4.43 \\
\hline 0.25 & 2.16 & 0.42 & 3.35 \\
\hline 0.20 & 1.68 & 0.32 & 2.44 \\
\hline \multicolumn{2}{|c|}{$\begin{aligned} \mathbf{k} & =9.1 \mathrm{M}^{-1} \mathrm{~s}^{-1} \\
\Delta \mathbf{k}^{a} & =0.8\end{aligned}$} & \multicolumn{2}{|c|}{$\begin{array}{l}\mathbf{k}=8.8 \mathrm{M}^{-1} \mathrm{~s}^{-1} \\
\mathbf{k}^{a}=0 ?\end{array}$} \\
\hline \multicolumn{2}{|c|}{$\mathbf{R}^{2}=0.9876$} & \multicolumn{2}{|c|}{$\mathbf{R}^{2}=0.9995$} \\
\hline
\end{tabular}

${ }^{a}$ calculated as the confidence interval of the slope for the $90 \%$ confidence level.

\begin{tabular}{cc}
\hline \multicolumn{2}{c}{$10 \mathrm{mM} \mathrm{CH}_{3} \mathrm{CO}_{2} \mathrm{H}$} \\
\hline$[\mathrm{CU}]$ & $\begin{array}{c}\mathrm{kx} \times 10^{3} \\
/ \mathrm{s}-1\end{array}$ \\
\hline 0.881 & 6.51 \\
0.685 & 5.12 \\
0.514 & 4.24 \\
0.367 & 3.29 \\
0.245 & 2.47 \\
0.147 & 1.63 \\
0.073 & 0.95
\end{tabular}

\begin{tabular}{cc}
\hline \multicolumn{2}{c}{$1000 \mathrm{mM} \mathrm{CH}_{3} \mathrm{CO}_{2} \mathrm{H}$} \\
\hline$[\mathrm{CU}]$ & $\mathrm{k}_{\mathrm{ex}} \times 10^{3}$ \\
$/ \mathrm{mM}-1$ \\
\hline 0.991 & 2.16 \\
0.771 & 1.71 \\
0.578 & 1.35 \\
0.413 & 1.12 \\
0.275 & 0.820 \\
0.165 & 0.612 \\
0.083 & 0.407
\end{tabular}

\begin{aligned} $\mathbf{k} & =6.7 \mathrm{M}^{-1} \mathrm{~s}^{-1} \\ \Delta \mathbf{k}^{a} & =0.4 \\$\hline $\mathbf{R}^{2} & =0.9942\end{aligned}$

\begin{aligned} $\mathbf{k} & =1.9 \mathrm{M}^{-1} \mathrm{~s}^{-1} \\ \Delta \mathbf{k}^{a} & =0.1 \\$\hline $\mathbf{R}^{2} & =0.9972\end{aligned}$

${ }^{a}$ calculated as the confidence interval of the slope for the $90 \%$ confidence level.

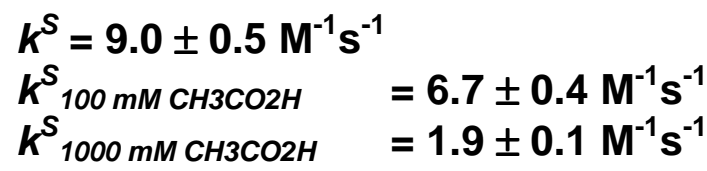


Table S16. Kinetic data for the reaction of $\mathbf{d p p h}^{\bullet}$ with $\mathrm{DHZ}$ in ethyl acetate and in ethyl acetate containing acetic acid. Symbols are the same as described in Table S1.

\begin{tabular}{|c|c|c|c|c|c|}
\hline \multicolumn{2}{|c|}{ neat ethyl acetate } & \multicolumn{2}{|c|}{$10 \mathrm{mM} \mathrm{CH}_{3} \mathrm{CO}_{2} \mathrm{H}$} & \multicolumn{2}{|c|}{$1000 \mathrm{mM} \mathrm{CH}_{3} \mathrm{CO}_{2} \mathrm{H}$} \\
\hline $\begin{array}{c}\text { [DHZ] } \\
/ \mathrm{mM}\end{array}$ & $\begin{array}{c}\mathrm{k}_{\mathrm{ex}} \times 10^{3} \\
/ \mathrm{s}-1\end{array}$ & $\begin{array}{c}{[\mathrm{DHZ}]} \\
/ \mathrm{mM} \\
\end{array}$ & $\begin{array}{c}\mathrm{k}_{\mathrm{ex}} \times 10^{3} \\
/ \mathrm{s}-1\end{array}$ & $\begin{array}{c}\mathrm{DHZ}] \\
/ \mathrm{mM}\end{array}$ & $\begin{array}{c}\mathrm{k}_{\mathrm{ex}} \times 10^{3} \\
/ \mathrm{s}-1\end{array}$ \\
\hline 1.72 & 1.29 & 1.72 & 1.34 & 1.34 & 1.03 \\
\hline 1.34 & 1.09 & 1.34 & 1.10 & 1.040 & 0.831 \\
\hline 1.04 & 0.823 & 1.04 & 0.926 & 0.780 & 0.651 \\
\hline 0.780 & 0.683 & 0.780 & 0.676 & 0.585 & 0.551 \\
\hline 0.585 & 0.485 & 0.585 & 0.625 & 0.439 & 0.476 \\
\hline 0.439 & 0.398 & 0.439 & 0.520 & 0.329 & 0.357 \\
\hline $\begin{array}{r}0.329 \\
\Delta \mathrm{l} \\
\mathbf{R}\end{array}$ & $\begin{array}{l}\quad 0.306 \\
.72 \mathrm{M}^{-1} \mathrm{~s}^{-1} \\
.05 \\
.9938\end{array}$ & 0.329 & $\begin{array}{l}0.405 \\
66 \mathrm{M}^{-1} \mathrm{~s}^{-1} \\
.05 \\
9926\end{array}$ & & $\begin{array}{l}.65 \mathrm{M}^{-1} \mathrm{~s}^{-1} \\
.04 \\
.9948\end{array}$ \\
\hline
\end{tabular}

${ }^{a}$ calculated as the confidence interval of the slope for the $90 \%$ confidence level.

$$
\begin{array}{ll}
\boldsymbol{k}^{S}=0.72 \pm 0.05 \mathrm{M}^{-1} \mathrm{~s}^{-1} \\
\boldsymbol{k}^{s}{ }_{10 \mathrm{mM} \mathrm{CH} 3 \mathrm{CO} 2 \mathrm{H}} & =0.66 \pm 0.05 \mathrm{M}^{-1} \mathrm{~s}^{-1} \\
\boldsymbol{k}_{1000 \mathrm{mM} \mathrm{CH}}^{s^{1} \mathrm{CO} 2 \mathrm{H}} & =0.65 \pm 0.04 \mathrm{M}^{-1} \mathrm{~s}^{-1}
\end{array}
$$


Table S17. Kinetic data for the reaction of $\mathrm{dpph}^{\bullet}$ with IE in ethyl acetate and in ethyl acetate containing acetic acid. Symbols are the same as described in Table S1.

\begin{tabular}{|c|c|c|c|}
\hline $\begin{array}{l}{[\mathrm{IE}]} \\
/ \mathrm{mM}\end{array}$ & $\begin{array}{c}\mathrm{k}_{\mathrm{ex}} \times 10^{3} \\
/ \mathrm{s}-1\end{array}$ & $\begin{array}{l}{[\mathrm{IE}]} \\
/ \mathrm{mM}\end{array}$ & $\begin{array}{c}\mathrm{k}_{\mathrm{ex}} \times 10^{3} \\
/ \mathrm{s}-1\end{array}$ \\
\hline 4.11 & 16.90 & 8.22 & 34.02 \\
\hline 3.20 & 13.34 & 6.39 & 26.76 \\
\hline 2.40 & 10.47 & 4.80 & 20.40 \\
\hline 1.71 & 7.57 & 3.43 & 14.74 \\
\hline 1.14 & 5.26 & 2.28 & 10.04 \\
\hline 0.69 & 3.29 & 1.37 & 6.26 \\
\hline 0.34 & 1.83 & 0.69 & 3.21 \\
\hline \multicolumn{2}{|c|}{$\begin{aligned} \mathbf{k} & =4.0 \mathrm{M}^{-1} \mathrm{~s}^{-1} \\
\Delta \mathbf{k}^{a} & =0.09 \\
\mathbf{R}^{2} & =0.9992\end{aligned}$} & \multicolumn{2}{|c|}{$\begin{aligned} \mathbf{k} & =4.1 \mathrm{M}^{-1} \mathrm{~s} \\
\Delta \mathbf{k}^{a} & =0.05 \\
\mathbf{R}^{2} & =0.9998\end{aligned}$} \\
\hline
\end{tabular}

${ }^{a}$ calculated as the confidence interval of the slope for the $90 \%$ confidence level.

\begin{tabular}{|c|c|c|c|}
\hline \multicolumn{2}{|c|}{$10 \mathrm{mM} \mathrm{CH}_{3} \mathrm{CO}_{2} \mathrm{H}$} & \multicolumn{2}{|c|}{$1000 \mathrm{mM} \mathrm{CH}_{3} \mathrm{CO}_{2} \mathrm{H}$} \\
\hline $\begin{array}{c}{[\mathrm{IE}]} \\
/ \mathrm{mM}\end{array}$ & $\begin{array}{c}\mathrm{k}_{\mathrm{ex}} \times 10^{3} \\
/ \mathrm{s}-1\end{array}$ & $\begin{array}{l}\text { [IE] } \\
/ \mathrm{mM}\end{array}$ & $\begin{array}{c}\mathrm{k}_{\mathrm{ex}} \times 10^{3} \\
/ \mathrm{s}-1\end{array}$ \\
\hline 15.1 & 66.4 & 18.8 & 85.8 \\
\hline 11.7 & 51.8 & 14.6 & 67.0 \\
\hline 8.79 & 39.2 & 11.0 & 51.0 \\
\hline 6.28 & 28.2 & 7.85 & 36.8 \\
\hline 4.19 & 19.4 & 5.23 & 24.8 \\
\hline 2.51 & 11.9 & 3.14 & 15.2 \\
\hline 1.26 & 7.60 & 1.57 & 9.44 \\
\hline \multicolumn{2}{|c|}{$\begin{aligned} \mathbf{k} & =4.3 \mathrm{M}^{-1} \mathrm{~s}^{-1} \\
\Delta \mathbf{k}^{a} & =0.06 \\
\mathbf{R}^{2} & =0.9997\end{aligned}$} & \multicolumn{2}{|c|}{$\begin{aligned} \mathbf{k} & =4.5 \mathrm{M}^{-1} \mathrm{~s}^{-1} \\
\Delta \mathbf{k}^{a} & =0.05 \\
\mathbf{R}^{2} & =0.9998\end{aligned}$} \\
\hline
\end{tabular}

$\bar{a}$ calculated as the confidence interval of the slope for the $90 \%$ confidence level.

$k^{S}=4.1 \pm 0.1 \mathrm{M}^{-1} \mathrm{~s}^{-1}$

$k_{10 \mathrm{mM} \mathrm{CH}}^{S_{10 O 2 H}}=4.3 \pm 0.1 \mathrm{M}^{-1} \mathrm{~s}^{-1}$

$k^{S}{ }_{1000 \mathrm{mM} \mathrm{CH} 3 \mathrm{CO} 2 \mathrm{H}}=4.5 \pm 0.1 \mathrm{M}^{-1} \mathrm{~s}^{-1}$ 

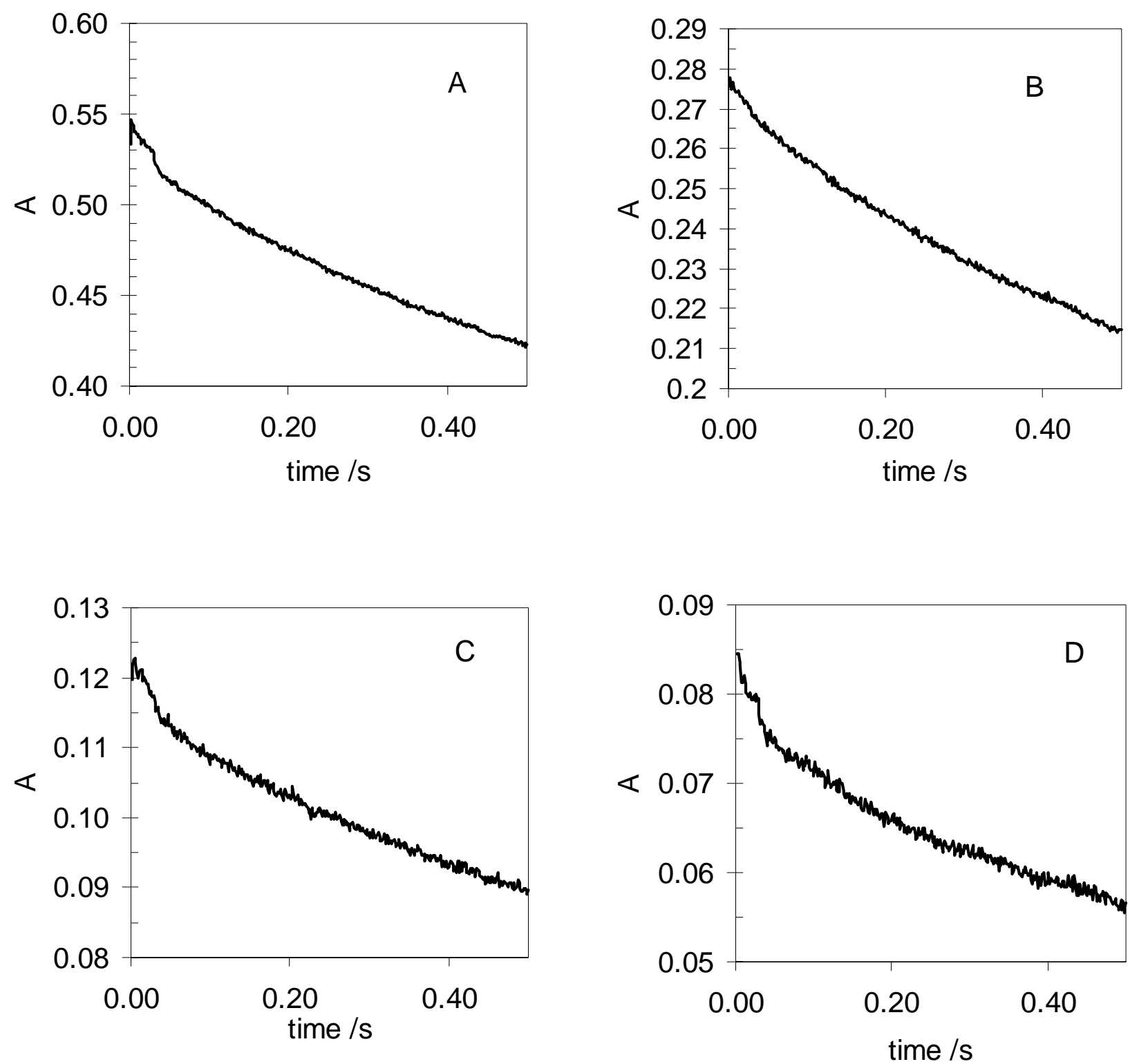

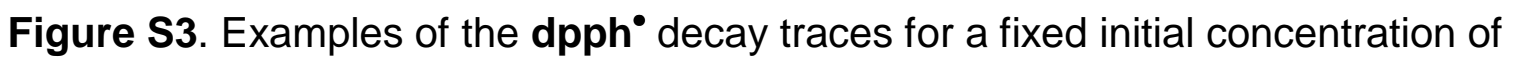
$\mathrm{DHZ}(0.98 \mathrm{mM})$ and variable initial concentrations of dpph ${ }^{\bullet}$ in methanol: A) $5.29 \times 10^{-5}$ $\left.\mathrm{M}, \mathrm{B}) 2.70 \times 10^{-5} \mathrm{M}, \mathrm{C}\right) 1.20 \times 10^{-5} \mathrm{M}$, and D) $0.80 \times 10^{-5} \mathrm{M}$. 
Table S18. Data used for kinetic estimation of the equilibrium concentration $\mathrm{DHZ}$ anion in methanol: $\left[\mathbf{d p p h}^{\circ}\right]_{0}=$ initial concentrations of dpph ${ }^{\bullet}, \Delta\left[\mathrm{dpph}^{\circ}\right]=\left[\mathbf{d p p h}{ }^{*}\right]_{0}$ [dpph $\left.{ }^{\circ}\right]_{\text {intercept. }}$

\begin{tabular}{cccc}
\hline No. & $\begin{array}{c}{\left[\text { dpph }^{\bullet}\right]_{0} \times 10^{5}} \\
/ \mathrm{M}\end{array}$ & $\begin{array}{c}1 /\left[\mathrm{dpph}^{\circ}\right]_{0} \times 10^{-4} \\
/ \mathrm{M}^{-1}\end{array}$ & $\begin{array}{c}\Delta\left[\mathrm{dpph}^{\circ}\right] \times 10^{-6} \\
/ \mathrm{M}\end{array}$ \\
\hline 1 & 9.07 & 1.10 & 2.53 \\
2 & 7.06 & 1.42 & 2.46 \\
3 & 5.29 & 1.89 & 2.40 \\
4 & 3.78 & 2.65 & 2.18 \\
5 & 2.70 & 3.70 & 1.98 \\
6 & 1.80 & 5.56 & 1.71 \\
7 & 1.20 & 8.33 & 1.46 \\
8 & 0.80 & 12.5 & 0.928 \\
\hline
\end{tabular}



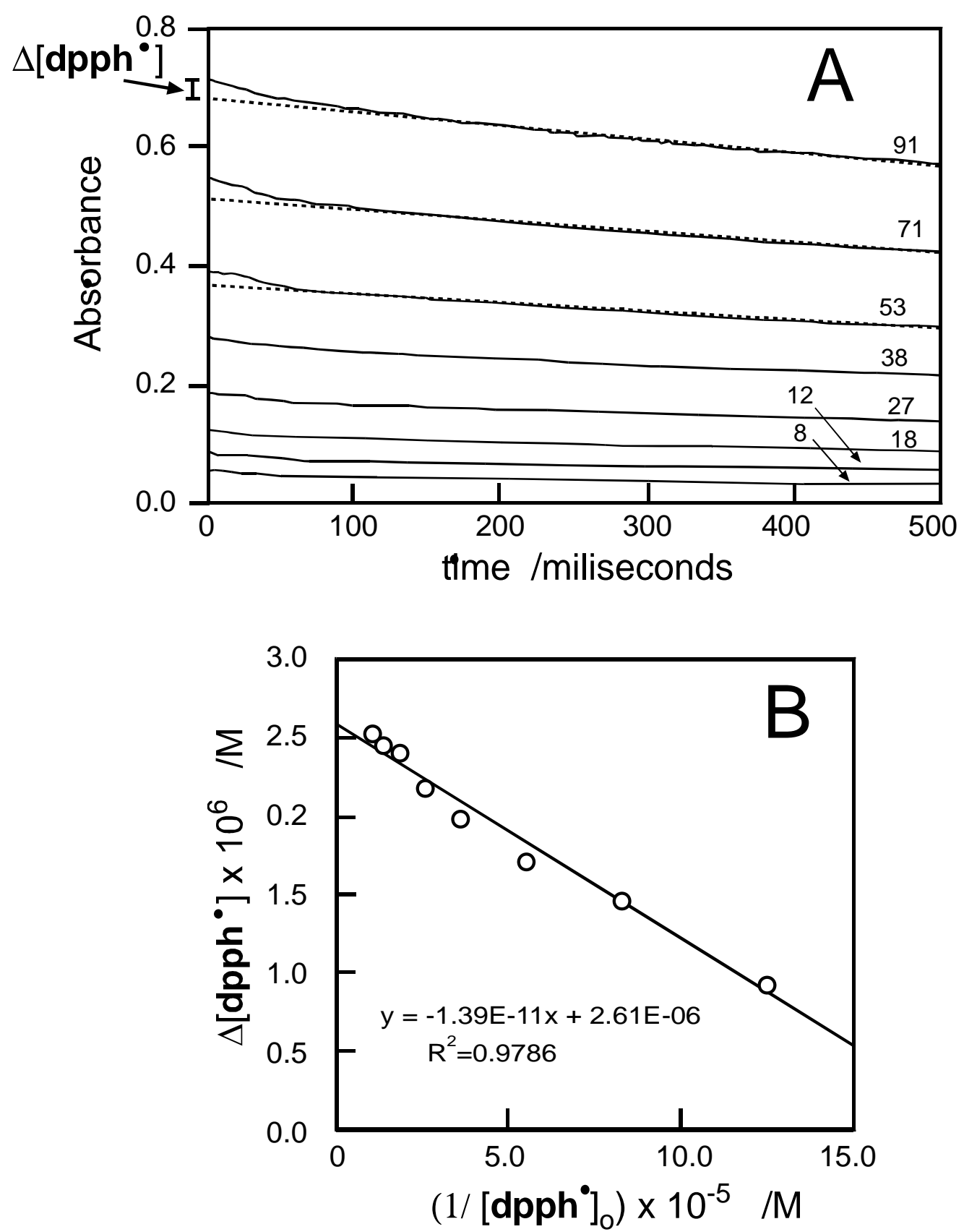

Figure S4. Panel A: Decay traces of dpph $^{-}$reacting with $\mathrm{DHZ}$ in methanol, $[\mathrm{DHZ}]=0.98 \mathrm{mM}=$ const. The annotations on the lines correspond to $10^{6}$ [dpph ${ }^{\circ}$ 。 $/ \mathrm{M}$, where [ $\left.\mathrm{dpph}^{\circ}\right]_{0}$ is the initial concentration. The determination of $\Delta\left[\mathbf{d p p h}^{\circ}\right]$ is shown for the higher $\left[\mathbf{d p p h}^{\circ}\right]_{0}$. Panel B: plot of [ $\left.\mathbf{d p p h}^{\circ}\right]_{0}-\left[\mathbf{d p p h}^{\circ}\right]_{\text {intercept }}=\Delta\left[\mathbf{d p p h}^{\circ}\right]$ as a function of reciprocal of [dpph $\left.{ }^{\circ}\right]_{0}$. 
Table S19. Kinetic data for the reaction of dpph ${ }^{\bullet}$ with $\mathrm{DHZ}$, 2-MeO-phenol, IE, and 4-Me-2-MeO-phenol in heptane. Symbols are the same as described in Table S1.

\begin{tabular}{rrrr}
\hline $\begin{array}{r}\mathrm{DHZ}] \\
/ \mathrm{mM}\end{array}$ & $\begin{array}{r}10^{3} \mathrm{k}_{\mathrm{ex}} \\
/ \mathrm{s}^{-1}\end{array}$ & $\begin{array}{r}{[\mathrm{DHZ}]} \\
/ \mathrm{mM}\end{array}$ & $\begin{array}{r}10^{3} \mathrm{k}_{\text {ex }} \\
/ \mathrm{s}^{-1}\end{array}$ \\
\hline 3.02 & 20.84 & 2.05 & 15.27 \\
2.35 & 17.33 & 1.60 & 12.08 \\
1.76 & 13.38 & 1.24 & 9.72 \\
1.26 & 9.52 & 0.93 & 7.13 \\
0.84 & 6.24 & 0.66 & 5.32 \\
0.56 & 4.52 & 0.44 & 3.54 \\
0.34 & 2.85 & 0.27 & 2.29 \\
0.20 & 2.10 & 0.16 & 1.33 \\
0.10 & 1.03 & 0.08 & 0.82
\end{tabular}

\begin{tabular}{rlrl}
$\mathbf{k}$ & $=6.9 \mathrm{M}^{-1} \mathrm{~s}^{-1}$ & $\mathbf{k}$ & $=7.4 \mathrm{M}^{-1} \mathrm{~s}^{-1}$ \\
$\Delta \mathbf{k}^{\mathbf{a}}$ & $=0.3 \mathrm{M}^{-1} \mathrm{~s}^{-1}$ & $\Delta \mathbf{k}^{\mathbf{a}}$ & $=0.1 \mathrm{M}^{-1} \mathrm{~s}^{-1}$ \\
$\mathbf{R}^{2}$ & $=0.9972$ & $\mathbf{R}^{2}$ & $=0.9993$ \\
\hline
\end{tabular}

\begin{tabular}{|c|c|c|c|c|c|}
\hline \multicolumn{2}{|c|}{ 2-MeO-phenol } & \multicolumn{2}{|c|}{ IE } & \multicolumn{2}{|c|}{ 4-Me-2-MeO-phenol } \\
\hline $\begin{array}{r}{[\mathrm{PhOH}]} \\
/ \mathrm{mM}\end{array}$ & $\begin{array}{r}10^{3} \mathrm{k}_{\mathrm{ex}} \\
/ \mathrm{s}^{-1}\end{array}$ & $\begin{array}{c}{[\mathrm{IE}]} \\
/ \mathrm{mM}\end{array}$ & $\begin{array}{r}10^{3} \mathrm{k}_{\mathrm{ex}} \\
/ \mathrm{s}^{-1}\end{array}$ & $\begin{array}{r}{[\mathrm{PhOH}]} \\
/ \mathrm{mM}\end{array}$ & $\begin{array}{c}10^{3} \mathrm{k}_{\mathrm{e}} \\
/ \mathrm{s}^{-1}\end{array}$ \\
\hline 12.37 & 12.8 & 4.35 & 230.3 & 12.5 & 91.7 \\
\hline 9.62 & 9.47 & 3.11 & 165.3 & 9.73 & 66.2 \\
\hline 7.22 & 7.35 & 2.07 & 103.4 & 7.30 & 51.7 \\
\hline 5.16 & 5.28 & 1.38 & 72.4 & 5.21 & 39.0 \\
\hline 3.44 & 3.61 & 0.92 & 47.1 & 3.47 & 27.7 \\
\hline 2.29 & 2.37 & 0.61 & 31.8 & 2.32 & 18.3 \\
\hline 1.53 & 1.68 & 0.41 & 21.3 & 1.54 & 13.2 \\
\hline 0.92 & 0.93 & 0.27 & 14.4 & 0.926 & 8.00 \\
\hline 0.55 & 0.57 & 0.18 & 10.0 & 0.556 & 5.10 \\
\hline \multirow[t]{3}{*}{0.27} & 0.29 & 0.11 & 6.24 & 0.334 & 2.98 \\
\hline & & & & 0.167 & 1.56 \\
\hline & & & & 0.083 & 0.751 \\
\hline \multicolumn{2}{|c|}{$\begin{aligned} \mathbf{k} & =1.01 \mathrm{M}^{-1} \mathrm{~s}^{-1} \\
\Delta \mathbf{k}^{\mathbf{a}} & =0.02 \mathrm{M}^{-1} \mathrm{~s}^{-1} \\
\mathbf{R}^{2} & =0.9990\end{aligned}$} & \multicolumn{2}{|c|}{$\begin{aligned} \mathbf{k} & =53 \mathrm{M}^{-1} \mathbf{s}^{-1} \\
\Delta \mathbf{k}^{\mathbf{a}} & =0.8 \mathrm{M}^{-1} \mathrm{~s}^{-1} \\
\mathbf{R}^{2} & =0.9993\end{aligned}$} & \multicolumn{2}{|c|}{$\begin{aligned} \mathbf{k} & =7.1 \mathrm{M}^{-1} \mathrm{~s}^{-1} \\
\Delta \mathbf{k}^{\mathbf{a}} & =0.2 \mathrm{M}^{-1} \mathrm{~s}^{-1} \\
\mathbf{R}^{2} & =0.9970\end{aligned}$} \\
\hline
\end{tabular}

$$
\begin{aligned}
k_{\mathrm{DHZ}}^{S} & =7.2 \pm 0.2 \mathrm{M}^{-1} \mathrm{~s}^{-1} \\
k_{\text {2-MeO-pheno }}^{S} & =1.0 \pm 0.1 \mathrm{M}^{-1} \mathrm{~s}^{-1} \\
k_{\text {IE }}^{S} & =53 \pm 0.8 \mathrm{M}^{-1} \mathrm{~s}^{-1} \\
k_{4-\mathrm{Me}-2-\mathrm{MeO} \text {-phenol }}^{S} & =7.1 \pm 0.2 \mathrm{M}^{-1} \mathrm{~s}^{-1}
\end{aligned}
$$


Table S20. Kinetic data for the reaction of $\mathrm{dpph}^{\bullet}$ with 2-MeO-phenol and 4-Me-2$\mathrm{MeO}-$ phenol in ethyl acetate. Symbols are the same as described in Table S1.

\begin{tabular}{|c|c|c|c|}
\hline \multicolumn{2}{|c|}{ 2-MeO-phenol } & \multicolumn{2}{|c|}{ 4-Me-2-MeO-phenol } \\
\hline $\begin{array}{r}{[\mathrm{PhOH}]} \\
/ \mathrm{mM}\end{array}$ & $\begin{array}{c}10^{3} \mathrm{k}_{\mathrm{ex}} \\
/ \mathrm{s}^{-1}\end{array}$ & $\begin{array}{r}{[\mathrm{PhOH}]} \\
/ \mathrm{mM}\end{array}$ & $\begin{array}{r}10^{3} \mathrm{k}_{\mathrm{ex}} \\
/ \mathrm{s}^{-1}\end{array}$ \\
\hline 39.96 & 5.43 & 18.64 & 16.77 \\
\hline 31.08 & 4.48 & 14.50 & 12.75 \\
\hline 23.31 & 2.80 & 10.88 & 9.85 \\
\hline 16.65 & 2.02 & 7.77 & 7.11 \\
\hline 11.10 & 1.39 & 5.18 & 4.81 \\
\hline 6.66 & 0.81 & 3.11 & 2.92 \\
\hline 3.33 & 0.40 & 1.55 & 1.45 \\
\hline 1.67 & 0.18 & 0.78 & 0.76 \\
\hline \multicolumn{2}{|c|}{$\begin{aligned} \mathbf{k} & =0.14 \mathrm{M}^{-1} \mathrm{~s}^{-1} \\
\Delta \mathbf{k}^{\mathbf{a}} & =0.01 \mathrm{M}^{-1} \mathrm{~s}^{-1} \\
\mathbf{R}^{2} & =0.9917\end{aligned}$} & \multicolumn{2}{|c|}{$\begin{aligned} \mathbf{k} & =0.89 \mathrm{M}^{-1} \mathrm{~s}^{-1} \\
\Delta \mathbf{k}^{\mathbf{a}} & =0.01 \mathrm{M}^{-1} \mathrm{~s}^{-1} \\
\mathbf{R}^{2} & =0.9996\end{aligned}$} \\
\hline
\end{tabular}

$k_{\text {2-MeO-phenol }}^{S} 0.14 \pm 0.01 \mathrm{M}^{-1} \mathrm{~s}^{-1}$

$k^{S}{ }_{4-M e-2-M e O-p h e n o l}=0.89 \pm 0.01 \mathrm{M}^{-1} \mathrm{~s}^{-1}$

Table S21. Kinetic data for the reaction of $\mathrm{dpph}^{\bullet}$ with 2-MeO-phenol and 4-Me-2MeO-phenol in dioxane. Symbols are the same as described in Table S1.

\begin{tabular}{|c|c|c|c|}
\hline \multicolumn{2}{|c|}{ 2-MeO-phenol } & \multicolumn{2}{|c|}{ 4-Me-2-MeO-phenol } \\
\hline $\begin{array}{r}{[\mathrm{PhOH}]} \\
/ \mathrm{mM}\end{array}$ & $\begin{array}{r}10^{3} \mathrm{k}_{\mathrm{ex}} \\
/ \mathrm{s}^{-1}\end{array}$ & $\begin{array}{r}{[\mathrm{PhOH}]} \\
/ \mathrm{mM}\end{array}$ & $\begin{array}{r}10^{3} \mathrm{k}_{\mathrm{ex}} \\
/ \mathrm{s}^{-1}\end{array}$ \\
\hline 34.42 & 1.92 & 20.01 & 9.65 \\
\hline 26.77 & 1.41 & 15.56 & 7.25 \\
\hline 20.08 & 1.10 & 11.67 & 5.72 \\
\hline 14.34 & 0.76 & 8.34 & 4.15 \\
\hline 9.56 & 0.50 & 5.56 & 2.84 \\
\hline 5.74 & 0.30 & 3.34 & 1.83 \\
\hline 2.87 & 0.18 & 1.67 & 0.87 \\
\hline \multirow[t]{2}{*}{1.43} & 0.08 & 0.83 & 0.48 \\
\hline & & 0.42 & 0.25 \\
\hline \multicolumn{2}{|c|}{$\begin{aligned} \mathbf{k} & =0.055 \\
\Delta \mathbf{k}^{\mathbf{a}} & =0.002 \\
\mathbf{R}^{2} & =0.9980\end{aligned}$} & \multicolumn{2}{|c|}{$\begin{aligned} \mathbf{k} & =0.47 \mathrm{M}^{-1} \mathrm{~s}^{-1} \\
\Delta \mathbf{k}^{a} & =0.01 \mathrm{M}^{-1} \mathrm{~s}^{-1} \\
\mathbf{R}^{2} & =0.9988\end{aligned}$} \\
\hline
\end{tabular}

$k^{S}{ }_{2-M e O-p h e n o l}=0.055 \pm 0.002 \mathrm{M}^{-1} \mathrm{~s}^{-1}$

$k^{S}{ }_{4-M e-2-M e O-p h e n o l}=0.47 \pm 0.01 \mathrm{M}^{-1} s^{-1}$ 
Table S22. Kinetic data for the reaction of dpph $^{\bullet}$ with acetylacetone in dioxane 24 hours after preparation of AcAc / dioxane solutions. Symbols are the same as described in Table S1.

\begin{tabular}{|c|c|}
\hline $\begin{array}{r}{[\mathrm{AcAc}]} \\
/ \mathrm{M}\end{array}$ & $\begin{array}{r}10^{5} \mathrm{k}_{\mathrm{ex}} \\
/ \mathrm{s}^{-1}\end{array}$ \\
\hline 2.02 & 10.19 \\
\hline 1.57 & 9.18 \\
\hline 1.22 & 6.68 \\
\hline 0.95 & 5.70 \\
\hline 0.63 & 4.20 \\
\hline \multicolumn{2}{|c|}{$\begin{aligned} \mathbf{k}= & 4.5 \times 10^{-5} \mathrm{M}^{-1} \mathrm{~s}^{-} \\
& 0.87 \times 10^{-5} \mathrm{M}^{-} \\
\Delta \mathbf{k}^{\mathbf{a}}= & =\mathrm{s}^{-1} \\
\mathbf{R}^{2}= & 0.9734\end{aligned}$} \\
\hline
\end{tabular}

$k^{S}{ }_{A c A C}=0.47 \pm 0.01 M^{-1} s^{-1}$

Table S23. Kinetic data for the reaction of $\mathbf{d p p h}^{\bullet}$ with AcAc in methanol freshly prepared and 24 hours after preparation of AcAc / methanol solutions. Symbols are the same as described in Table S1.

\begin{tabular}{|c|c|c|c|}
\hline \multicolumn{2}{|c|}{ fresh } & \multicolumn{2}{|c|}{24 hours after preparation } \\
\hline $\begin{array}{c}{[\mathrm{AcAc}]} \\
/ \mathrm{mM}\end{array}$ & $\begin{array}{r}10^{3} \mathrm{k}_{\mathrm{ex}} \\
/ \mathrm{s}^{-1}\end{array}$ & $\begin{array}{r}{[\mathrm{AcAc}]} \\
/ \mathrm{mM}\end{array}$ & $\begin{array}{l}10^{3} \mathrm{k}_{\mathrm{ex}} \\
/ \mathrm{s}^{-1}\end{array}$ \\
\hline 34.24 & 1.35 & 29.98 & 1.10 \\
\hline 26.63 & 1.05 & 23.32 & 0.79 \\
\hline 19.97 & 0.72 & 17.49 & 0.61 \\
\hline 14.27 & 0.55 & 12.49 & 0.43 \\
\hline 9.51 & 0.35 & 8.33 & 0.28 \\
\hline 5.71 & 0.26 & 5.00 & 0.20 \\
\hline 3.42 & 0.17 & 2.50 & 0.11 \\
\hline 1.71 & 0.09 & & \\
\hline \multicolumn{2}{|c|}{$\begin{aligned} \mathbf{k} & =0.038 \mathrm{M}^{-1} \mathrm{~s}^{-1} \\
\Delta \mathbf{k}^{\mathbf{a}} & =0.002 \mathrm{M}^{-1} \mathrm{~s}^{-1} \\
\mathbf{R}^{2} & =0.9955\end{aligned}$} & \multicolumn{2}{|c|}{$\begin{aligned} \mathbf{k} & =0.035 \mathrm{M}^{-1} \mathrm{~s}^{-1} \\
\Delta \mathbf{k}^{\mathbf{a}} & =0.002 \mathrm{M}^{-1} \mathrm{~s}^{-1} \\
\mathbf{R}^{2} & =0.9950\end{aligned}$} \\
\hline
\end{tabular}

$k_{\text {fresh }}^{s_{\text {fo }}}=0.038 \pm 0.002 \mathrm{M}^{-1} \mathrm{~s}^{-1}$
$k_{\text {old }}^{s^{\prime}}=0.035 \pm 0.002 \mathrm{M}^{-1} \mathrm{~s}^{-1}$ 
Figure S5. IR spectra of $14 \mathrm{mM}$ isoeugenol in $\mathrm{CCl} 4$ containing various concentrations of DMSO (values in $\mathrm{mM}$ ).

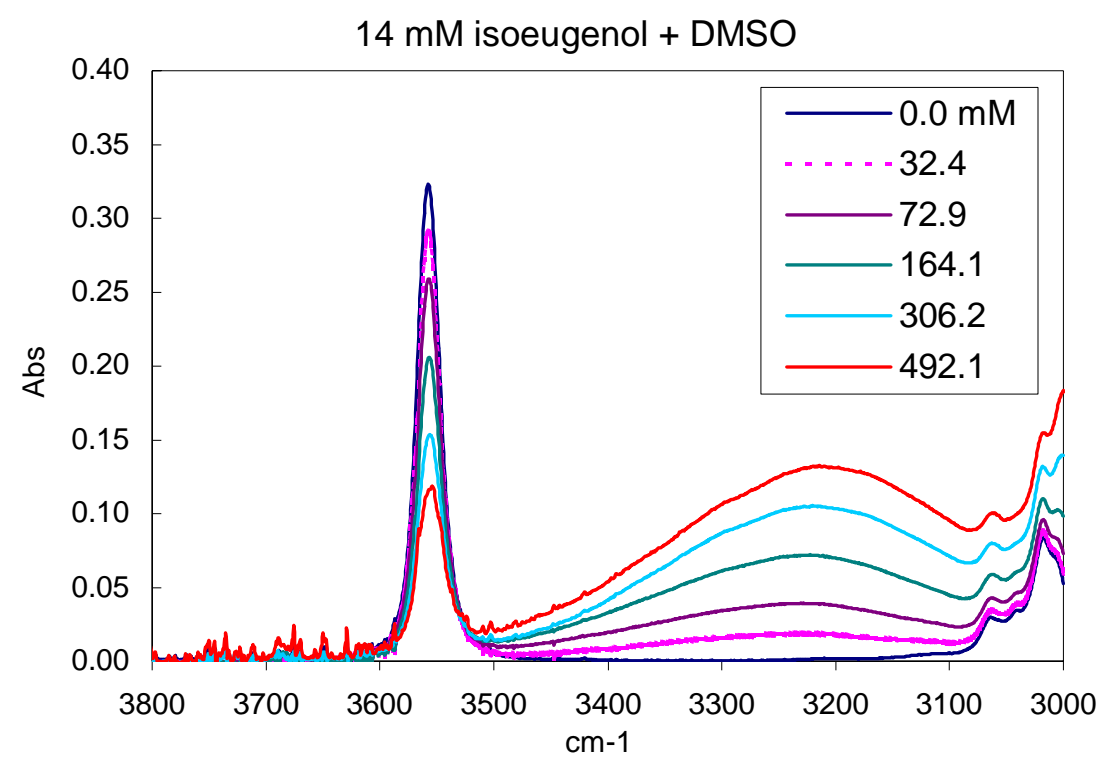

Figure S6. IR spectra of $8.7 \mathrm{mM} \mathrm{DHZ}$ in $\mathrm{CCl} 4$ containing various concentrations of DMSO (values in $\mathrm{mM}$ ).

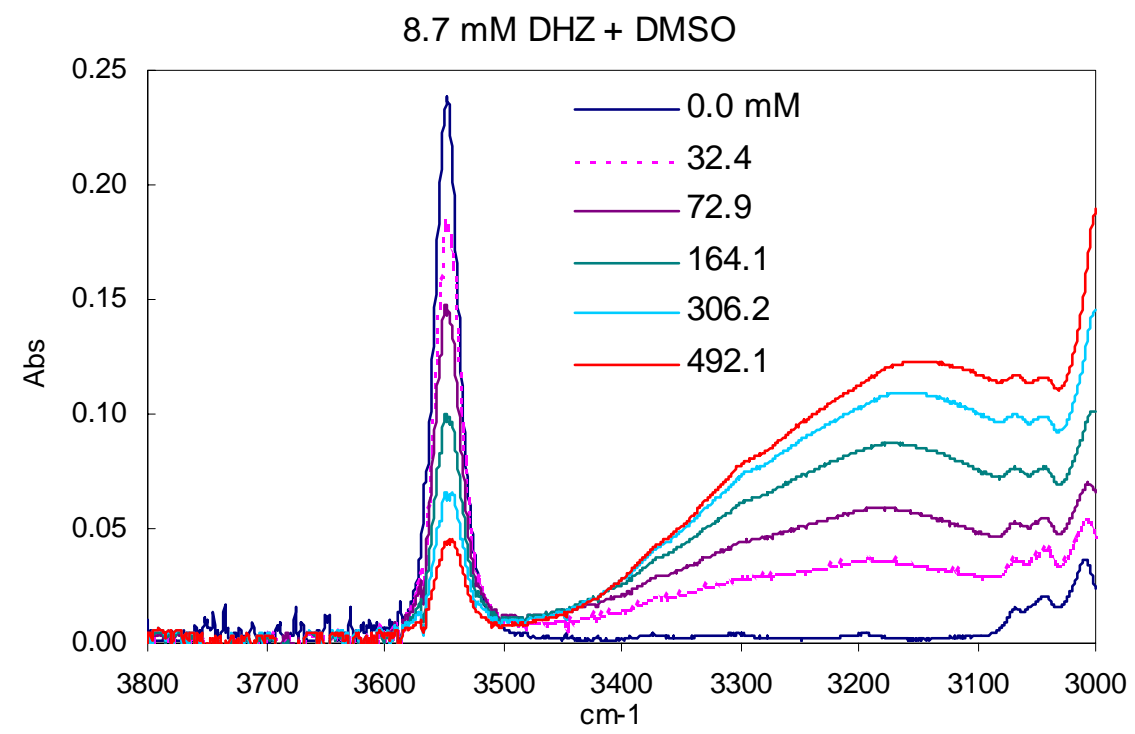


Table S24. Parameters used for calculation of the equilibrium constant $K_{\text {ArOH/S }}^{S}$ for HB complex formation between DMSO and four phenols. $[\mathrm{ArOH}]_{0}=$ total concentration of phenol, $[\mathrm{ArOH}]_{\text {free }}=$ concentration of non-hydrogen bonded phenol calculated from IR measurements, $[\mathrm{HB}]=[\mathrm{ArOH}]_{\mathrm{o}}$ $[\mathrm{ArOH}]_{\text {free }},[\mathrm{DMSO}]_{0}=$ total concentration of $\mathrm{DMSO}$ in $\mathrm{CCl}_{4},[\mathrm{DMSO}]_{\text {free }}=$ concentration of free (i.e. nonhydrogen bonded) DMSO. Plots of $[\mathrm{ArOH}]_{0} /[\mathrm{ArOH}]_{\text {free }}$ vs. $[\mathrm{DMSO}]_{\text {free }}$ are presented in Figure $\mathrm{S} 7$.

\begin{tabular}{|c|c|c|c|c|c|}
\hline$[\mathrm{ArOH}]_{0}$ & {$[\mathrm{ArOH}]_{\text {free }}$} & {$[\mathrm{HB}]$} & [DMSO] & {$[\mathrm{DMSO}]_{\text {free }}$} & {$[\mathrm{ArOH}]_{\mathrm{o}} /[\mathrm{ArOH}]_{\text {free }}$} \\
\hline \multicolumn{6}{|c|}{ DMSO + 2-methoxyphenol } \\
\hline 26.3 & 26.30 & 0.0 & 0.0 & 0.00 & 1.00 \\
\hline 26.3 & 23.07 & 3.2 & 24.5 & 21.26 & 1.14 \\
\hline 26.3 & 21.40 & 4.9 & 49.2 & 44.30 & 1.23 \\
\hline 26.3 & 18.71 & 7.6 & 98.4 & 90.81 & 1.41 \\
\hline 26.3 & 15.67 & 10.6 & 164.0 & 153.36 & 1.68 \\
\hline 26.3 & 13.55 & 12.8 & 273.3 & 260.55 & 1.94 \\
\hline \multicolumn{6}{|c|}{ DMSO + 2-methoxyphenol } \\
\hline 15.26 & 15.260 & 0.00 & 0.00 & 0.00 & 1.000 \\
\hline 15.26 & 13.989 & 1.27 & 19.40 & 18.13 & 1.091 \\
\hline 15.26 & 13.946 & 1.31 & 32.40 & 31.09 & 1.094 \\
\hline 15.26 & 12.794 & 2.47 & 48.60 & 46.13 & 1.193 \\
\hline 15.26 & 11.887 & 3.37 & 72.90 & 69.53 & 1.284 \\
\hline 15.26 & 10.701 & 4.56 & 109.40 & 104.84 & 1.426 \\
\hline 15.26 & 9.230 & 6.03 & 164.05 & 158.02 & 1.653 \\
\hline 15.26 & 7.819 & 7.44 & 229.70 & 222.26 & 1.952 \\
\hline 15.26 & 6.608 & 8.65 & 306.20 & 297.55 & 2.309 \\
\hline 15.26 & 5.657 & 9.60 & 393.70 & 384.10 & 2.698 \\
\hline \multicolumn{6}{|c|}{ DMSO + 4-Me-2-MeO-phenol } \\
\hline 14.31 & 14.311 & 0.000 & 0 & 0.000 & 1.000 \\
\hline 14.31 & 13.020 & 1.291 & 19.4 & 18.109 & 1.099 \\
\hline 14.31 & 12.612 & 1.699 & 32.4 & 30.701 & 1.135 \\
\hline 14.31 & 12.020 & 2.291 & 48.6 & 46.309 & 1.191 \\
\hline 14.31 & 11.209 & 3.102 & 72.9 & 69.798 & 1.277 \\
\hline 14.31 & 10.204 & 4.107 & 109.4 & 105.293 & 1.403 \\
\hline 14.31 & 8.917 & 5.394 & 164.05 & 158.656 & 1.605 \\
\hline 14.31 & 7.776 & 6.536 & 229.7 & 223.164 & 1.841 \\
\hline 14.31 & 6.505 & 7.806 & 306.2 & 298.394 & 2.200 \\
\hline 14.31 & 5.597 & 8.714 & 393.7 & 384.986 & 2.557 \\
\hline 14.31 & 4.740 & 9.571 & 492.1 & 482.529 & 3.019 \\
\hline \multicolumn{6}{|c|}{ DMSO + 4-Me-2-MeO-phenol } \\
\hline 13.35 & 0.282 & 13.347 & 0.000 & 0 & 0.000 \\
\hline 13.35 & 0.262 & 12.357 & 0.990 & 19.4 & 18.410 \\
\hline 13.35 & 0.255 & 12.015 & 1.332 & 32.4 & 31.068 \\
\hline 13.35 & 0.245 & 11.480 & 1.867 & 48.6 & 46.733 \\
\hline 13.35 & 0.231 & 10.755 & 2.592 & 72.9 & 70.308 \\
\hline 13.35 & 0.213 & 9.857 & 3.490 & 109.4 & 105.910 \\
\hline 13.35 & 0.190 & 8.694 & 4.653 & 164.1 & 159.397 \\
\hline 13.35 & 0.171 & 7.684 & 5.663 & 229.7 & 224.037 \\
\hline 13.35 & 0.147 & 6.505 & 6.842 & 306.2 & 299.358 \\
\hline 13.35 & 0.130 & 5.597 & 7.750 & 393.7 & 385.950 \\
\hline
\end{tabular}


Table S24. Continued

\begin{tabular}{|c|c|c|c|c|c|}
\hline$[\mathrm{ArOH}]_{0}$ & {$[\mathrm{ArOH}]_{\text {free }}$} & {$[\mathrm{HB}]$} & {$[\mathrm{DMSO}]_{0}$} & {$[\mathrm{DMSO}]_{\text {free }}$} & {$[\mathrm{ArOH}]_{0} /[\mathrm{ArOH}]_{\text {free }}$} \\
\hline \multicolumn{6}{|c|}{ DMSO + isoeugenol } \\
\hline 14.20 & 14.20 & 0.00 & 0.00 & 0.00 & 1.000 \\
\hline 14.20 & 13.29 & 0.91 & 19.40 & 18.49 & 1.069 \\
\hline 14.20 & 12.79 & 1.40 & 32.40 & 31.00 & 1.110 \\
\hline 14.20 & 12.19 & 2.01 & 48.60 & 46.59 & 1.165 \\
\hline 14.20 & 11.36 & 2.84 & 72.90 & 70.06 & 1.250 \\
\hline 14.20 & 10.29 & 3.91 & 109.40 & 105.49 & 1.380 \\
\hline 14.20 & 8.98 & 5.22 & 164.05 & 158.83 & 1.581 \\
\hline 14.20 & 7.71 & 6.49 & 229.70 & 223.21 & 1.841 \\
\hline 14.20 & 6.64 & 7.56 & 306.20 & 298.64 & 2.138 \\
\hline 14.20 & 5.75 & 8.44 & 393.70 & 385.26 & 2.467 \\
\hline 14.20 & 5.11 & 9.09 & 492.10 & 483.01 & 2.780 \\
\hline \multicolumn{6}{|c|}{$\mathrm{DMSO}+\mathrm{DHZ}$} \\
\hline 8.22 & 8.22 & 0.00 & 0.0 & 0.0 & 1.00 \\
\hline 8.22 & 6.86 & 1.36 & 19.4 & 18.0 & 1.20 \\
\hline 8.22 & 6.28 & 1.94 & 32.4 & 30.5 & 1.31 \\
\hline 8.22 & 5.66 & 2.56 & 48.6 & 46.0 & 1.45 \\
\hline 8.22 & 4.98 & 3.24 & 72.9 & 69.7 & 1.65 \\
\hline 8.22 & 4.20 & 4.02 & 109.4 & 105.4 & 1.96 \\
\hline 8.22 & 3.37 & 4.85 & 164.1 & 159.2 & 2.44 \\
\hline 8.22 & 2.68 & 5.55 & 229.7 & 224.2 & 3.07 \\
\hline 8.22 & 2.21 & 6.01 & 306.2 & 300.2 & 3.72 \\
\hline 8.22 & 1.72 & 6.50 & 393.7 & 387.2 & 4.79 \\
\hline 8.22 & 1.37 & 6.85 & 492.1 & 485.2 & 6.00 \\
\hline \multicolumn{6}{|c|}{$\mathrm{DMSO}+\mathrm{DHZ}$} \\
\hline 8.68 & 7.54 & 1.15 & 19.4 & 18.3 & 1.15 \\
\hline 8.68 & 6.91 & 1.78 & 32.4 & 30.6 & 1.26 \\
\hline 8.68 & 6.26 & 2.43 & 48.6 & 46.2 & 1.39 \\
\hline 8.68 & 5.51 & 3.18 & 72.9 & 69.7 & 1.58 \\
\hline 8.68 & 4.64 & 4.05 & 109.4 & 105.4 & 1.87 \\
\hline 8.68 & 3.68 & 5.00 & 164.1 & 159.0 & 2.36 \\
\hline 8.68 & 2.98 & 5.70 & 229.7 & 224.0 & 2.91 \\
\hline 8.68 & 2.38 & 6.30 & 306.2 & 299.9 & 3.65 \\
\hline 8.68 & 2.00 & 6.69 & 393.7 & 387.0 & 4.35 \\
\hline 8.68 & 1.59 & 7.09 & 492.1 & 485.0 & 5.45 \\
\hline
\end{tabular}


Figure S7. Plots of $[\mathrm{ArOH}]_{0} /[\mathrm{ArOH}]_{\text {free }}$ vs. [DMSO $]_{\text {free }}$ phenols listed in Table S24. The slopes of the constructed straight lines are the HB equilibrium constants

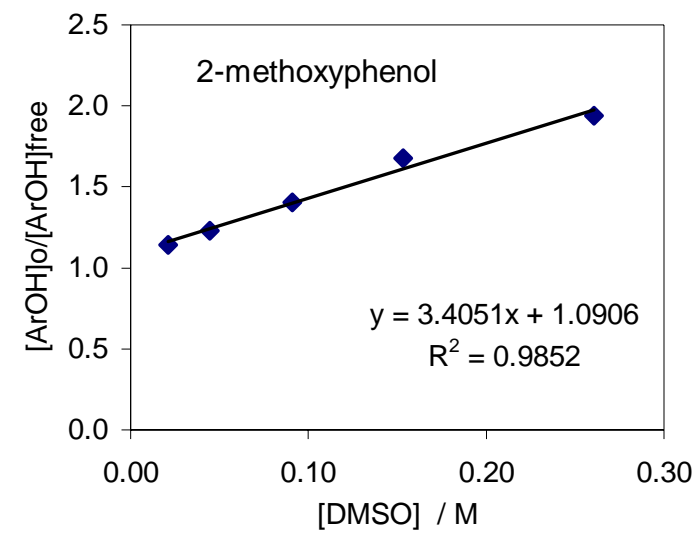

a)

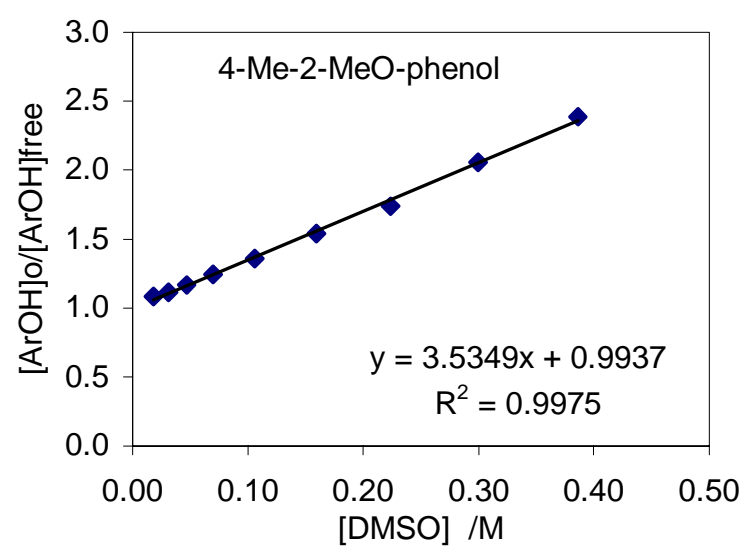

c)

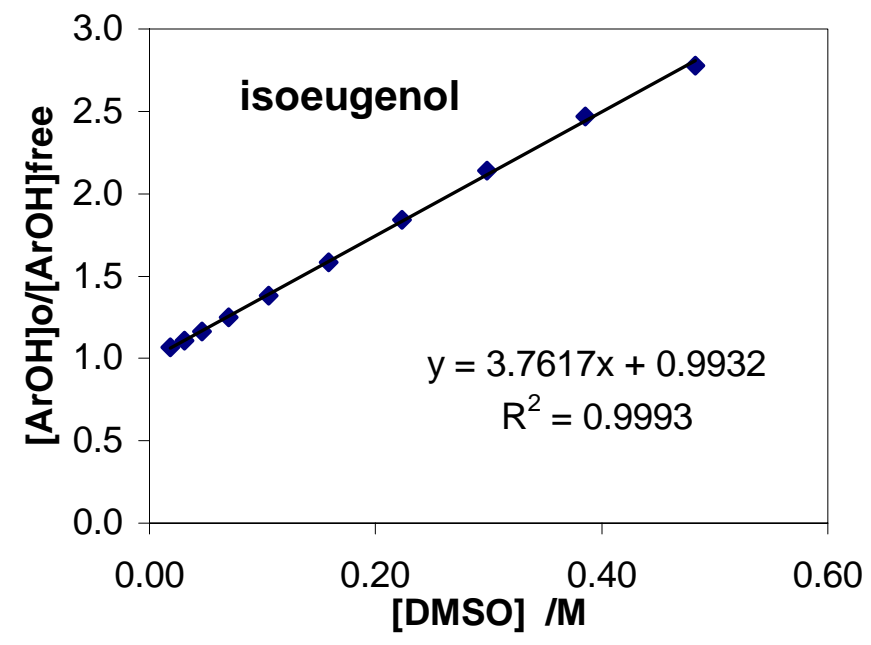

e)

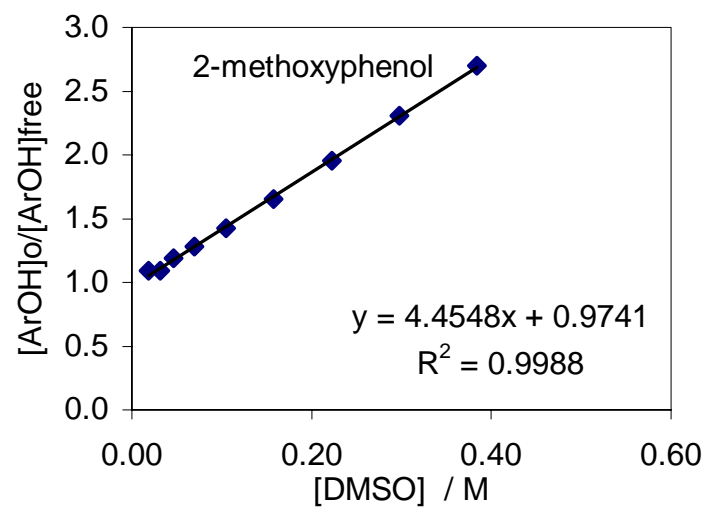

b)

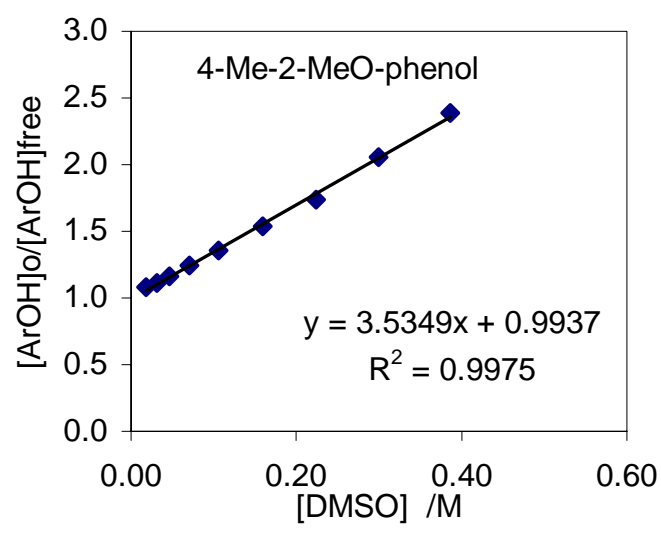

d) 
Figure S7. continued
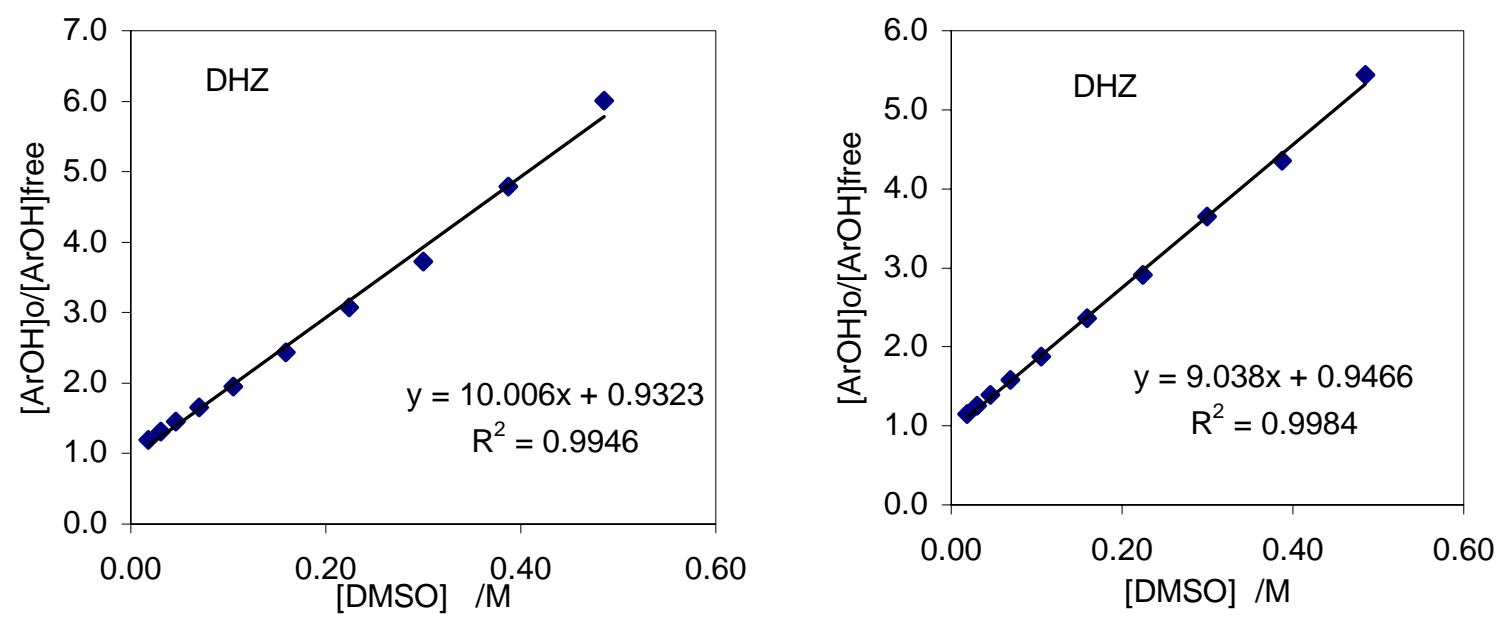

f)

g)

Table S25. Values $K_{A}^{H_{i}}, \log K_{A}^{H_{i}}$, and $\alpha_{2}^{H}$ for studied ortho-methoxyphenols calculated from IR measurements of $H B$ complex formation with DMSO $\left(L_{B}=1.24, D_{B}=0.266\right)$.

\begin{tabular}{lccc}
\hline phenol & $K_{A}^{H_{i}}$ & $\log K_{A}^{H_{i}}$ & $\alpha_{2}^{H}$ \\
\hline 2-MeO-phenol & 3.40 & 0.214 & 0.284 \\
2-MeO-phenol & 4.45 & 0.309 & 0.304 \\
4-Me-2-MeO-phenol & 4.10 & 0.280 & 0.298 \\
4-Me-2-MeO-phenol & 3.53 & 0.228 & 0.286 \\
IE & 3.76 & 0.250 & 0.291 \\
DHZ & 10.06 & 0.594 & 0.365 \\
DHZ & 9.04 & 0.557 & 0.357 \\
\hline
\end{tabular}


Table S26. Parameters used for calculation of the equilibrium constant $K_{\mathrm{ArOH} / \mathrm{S}}^{S_{\text {for }}}$ HB complex formation between 1,4-dioxane and two phenols. $[\mathrm{ArOH}]_{0}=$ total concentration of phenol, $[\mathrm{ArOH}]_{\text {free }}=$ concentration of non-hydrogen bonded phenol calculated from IR measurements, $[\mathrm{HB}]=[\mathrm{ArOH}]_{0}-[\mathrm{ArOH}]_{\text {free }}$, [dioxane $]_{0}=$ total concentration of dioxane in $\mathrm{CCl}_{4}$, [dioxane] free $=$ concentration of free (i.e. nonhydrogen bonded) dioxane. Plots of $[\mathrm{ArOH}]_{0} /[\mathrm{ArOH}]_{\text {free }}$ vs. [dioxane $]_{\text {free }}$ are presented in Figure S8.

\begin{tabular}{|c|c|c|c|c|c|}
\hline$[\mathrm{ArOH}]_{0}$ & {$[\mathrm{ArOH}]_{\text {free }}$} & {$[\mathrm{HB}]$} & [dioxane & [dioxane] free & \multirow{2}{*}[\mathrm{ArOH}]{$_{0} /[\mathrm{ArOH}]_{\text {free }}$} \\
\hline \multirow{2}{*}{\multicolumn{6}{|c|}{ 4-fluorophenol + dioxane }} \\
\hline 10.01 & & & & & \\
\hline 10.01 & 9.72 & 0.3 & 8.5 & 8.21 & 1.03 \\
\hline 10.01 & 8.96 & 1.0 & 16.9 & 15.85 & 1.12 \\
\hline 10.01 & 8.14 & 1.9 & 28.2 & 26.28 & 1.23 \\
\hline 10.01 & 7.06 & 2.9 & 46.9 & 43.95 & 1.42 \\
\hline 10.01 & 5.80 & 4.2 & 78.2 & 73.99 & 1.73 \\
\hline 10.01 & 4.34 & 5.7 & 130.4 & 124.73 & 2.31 \\
\hline 10.01 & 3.24 & 6.8 & 195.6 & 188.83 & 3.09 \\
\hline 10.01 & 2.48 & 7.5 & 273.8 & 266.28 & 4.03 \\
\hline \multicolumn{6}{|c|}{ 4-fluorophenol + dioxane } \\
\hline 8.66 & 8.66 & 0.0 & 0.0 & 0.00 & 1.00 \\
\hline 8.66 & 8.53 & 0.1 & 8.5 & 8.37 & 1.02 \\
\hline 8.66 & 7.94 & 0.7 & 16.9 & 16.17 & 1.09 \\
\hline 8.66 & 7.23 & 1.4 & 28.2 & 26.72 & 1.20 \\
\hline 8.66 & 6.26 & 2.4 & 46.9 & 44.50 & 1.38 \\
\hline 8.66 & 5.14 & 3.5 & 78.2 & 74.68 & 1.69 \\
\hline 8.66 & 3.92 & 4.7 & 130.4 & 125.66 & 2.21 \\
\hline 8.66 & 2.95 & 5.7 & 195.6 & 189.88 & 2.94 \\
\hline 8.66 & 2.28 & 6.4 & 273.8 & 267.41 & 3.81 \\
\hline 8.66 & 1.73 & 6.9 & 365.1 & 358.17 & 5.00 \\
\hline 8.66 & 1.35 & 7.3 & 469.4 & 462.09 & 6.40 \\
\hline \multicolumn{6}{|c|}{ 2-MeO-phenol + dioxane } \\
\hline 20.85 & 20.85 & 0.000 & 0 & 0.000 & 1.000 \\
\hline 20.85 & 20.76 & 0.086 & 24 & 23.914 & 1.004 \\
\hline 20.85 & 20.45 & 0.395 & 35.2 & 34.805 & 1.019 \\
\hline 20.85 & 20.09 & 0.755 & 58.7 & 57.945 & 1.038 \\
\hline 20.85 & 19.57 & 1.275 & 97.8 & 96.525 & 1.065 \\
\hline 20.85 & 18.62 & 2.222 & 163 & 160.778 & 1.119 \\
\hline 20.85 & 16.71 & 4.137 & 326 & 321.863 & 1.248 \\
\hline 20.85 & 15.14 & 5.712 & 489 & 483.288 & 1.377 \\
\hline 20.85 & 13.46 & 7.387 & 684.5 & 677.113 & 1.549 \\
\hline 20.85 & 11.90 & 8.943 & 912.7 & 903.757 & 1.751 \\
\hline 20.85 & 10.27 & 10.576 & 1173.5 & 1162.924 & 2.030 \\
\hline
\end{tabular}


Figure S8. Plots of $[\mathrm{ArOH}]_{\mathrm{o}} /[\mathrm{ArOH}]_{\text {free }}$ vs. [dioxane $]_{\text {free }}$ for phenols listed in Table S26. The slopes of the constructed straight lines are the HB equilibrium constants.

4-fluorophenol in dioxane

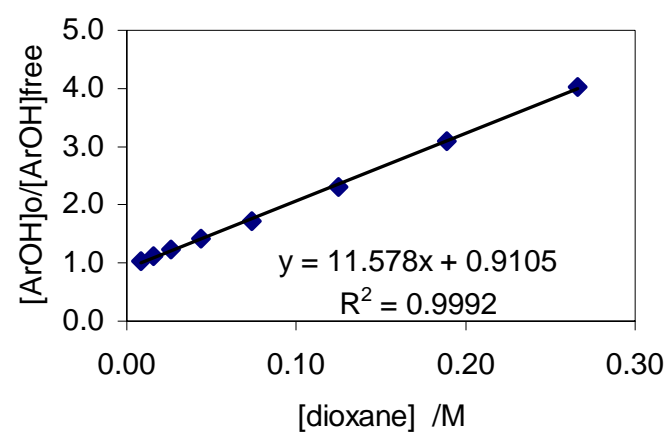

2-MeO-phenol + dioxane

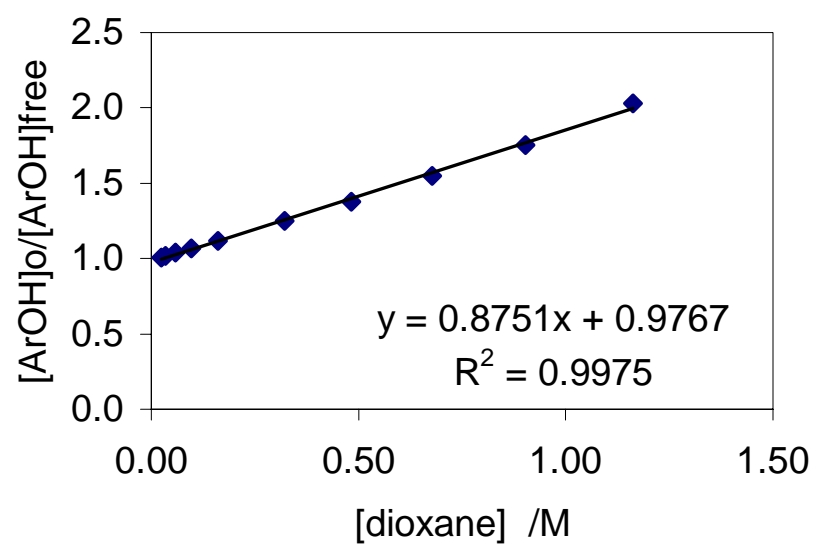

4-fluorophenol in dioxane

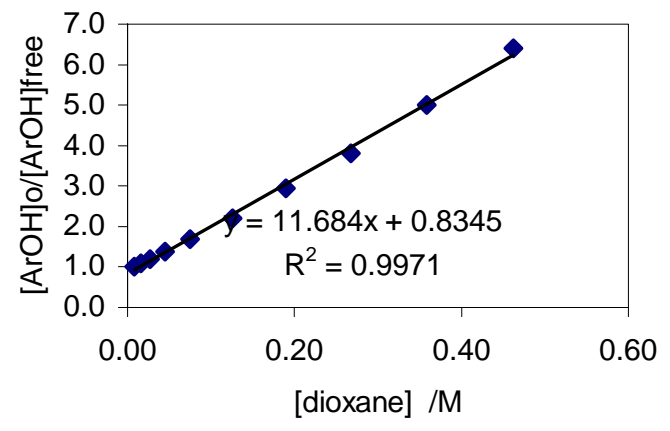


Table S27. Determination of ionization constant of 2-MeO-phenol $(85.0 \mathrm{mg}, 0.685$ $\mathrm{mmol}$ ) dissolved in $48 \mathrm{~mL}$ of methanol-water $(1: 1, \mathrm{v} / \mathrm{v}), 25^{\circ} \mathrm{C}$.

\begin{tabular}{|c|c|c|c|c|c|c|c|}
\hline 1 & 2 & 3 & 4 & 5 & 6 & 7 & 8 \\
\hline $\begin{array}{c}\text { Titrant } \\
0.0684 \mathrm{M} \\
\mathrm{KOH}\end{array}$ & $\mathrm{pH}$ & $\begin{array}{l}\text { Concentration } \\
\text { of phenol } \\
\text { [PhOH] }\end{array}$ & $\begin{array}{c}{[\mathrm{PhOH}]_{\circ}} \\
\text { minus } \\
\text { column } 3\end{array}$ & $\begin{array}{l}\text { activity } \\
\{\mathrm{OH}\}^{*}\end{array}$ & $\begin{array}{c}\text { Columns }(3+5) \\
\text { divided by } \\
\text { columns } \\
(4-5)\end{array}$ & $\begin{array}{l}\log \text { of } \\
\text { Column } \\
6\end{array}$ & $\begin{array}{c}\mathrm{p} K_{\mathrm{a}} \\
\text { (columns } \\
2+7 \text { ) }\end{array}$ \\
\hline$(\mathrm{mL})$ & & $\mathrm{M}$ & $\mathrm{M}$ & & & & \\
\hline 0.00 & 6.11 & 0.0141 & 0.000 & & & & \\
\hline 0.50 & 9.37 & 0.0133 & 0.001 & $1.58 \mathrm{E}-05$ & 19.49 & 1.29 & 10.66 \\
\hline 1.00 & 9.65 & 0.0125 & 0.001 & 3.02E-05 & 9.23 & 0.97 & 10.62 \\
\hline 1.50 & 9.85 & 0.0116 & 0.002 & 4.79E-05 & 5.83 & 0.77 & 10.62 \\
\hline 2.00 & 10.02 & 0.0108 & 0.003 & 7.08E-05 & 4.14 & 0.62 & 10.64 \\
\hline 2.50 & 10.15 & 0.0101 & 0.003 & $9.55 \mathrm{E}-05$ & 3.12 & 0.49 & 10.64 \\
\hline 3.00 & 10.27 & 0.0093 & 0.004 & $1.26 \mathrm{E}-04$ & 2.45 & 0.39 & 10.66 \\
\hline 3.50 & 10.39 & 0.0086 & 0.005 & 1.66E-04 & 1.97 & 0.29 & 10.68 \\
\hline 4.00 & 10.47 & 0.0078 & 0.005 & 2.00E-04 & 1.60 & 0.20 & 10.67 \\
\hline 4.50 & 10.58 & 0.0071 & 0.006 & 2.57E-04 & 1.33 & 0.12 & 10.70 \\
\hline 5.00 & 10.68 & 0.0064 & 0.006 & 3.24E-04 & 1.11 & 0.04 & 10.72 \\
\hline 5.50 & 10.77 & 0.0057 & 0.007 & $3.98 \mathrm{E}-04$ & 0.93 & -0.03 & 10.74 \\
\hline 6.00 & 10.87 & 0.0050 & 0.008 & 5.01E-04 & 0.79 & -0.10 & 10.77 \\
\hline 6.50 & 10.94 & 0.0044 & 0.008 & 5.89E-04 & 0.66 & -0.18 & 10.76 \\
\hline 7.00 & 11.02 & 0.0037 & 0.009 & 7.08E-04 & 0.56 & -0.25 & 10.77 \\
\hline 7.50 & 11.09 & 0.0031 & 0.009 & 8.32E-04 & 0.47 & -0.33 & 10.76 \\
\hline
\end{tabular}

Result: $\mathrm{p} K_{\mathrm{a}}=\mathbf{1 0 . 6 9} \pm \mathbf{0 . 0 8}$ at $0.014 \mathrm{M}$ and $25^{\circ} \mathrm{C}$ 
Table S28. Determination of ionization constant of 2-MeO-phenol $(86.2 \mathrm{mg}, 0.694$ $\mathrm{mmol})$ dissolved in $47 \mathrm{~mL}$ of methanol-water $(1: 1, \mathrm{v} / \mathrm{v}), 25^{\circ} \mathrm{C}$.

\begin{tabular}{cccccccc}
\hline $\mathbf{1}$ & $\mathbf{2}$ & $\mathbf{3}$ & $\mathbf{4}$ & $\mathbf{5}$ & $\mathbf{6}$ & $\mathbf{7}$ & $\mathbf{8}$ \\
\hline $\begin{array}{c}\text { Titrant } \\
0.100 \mathrm{M} \mathrm{KOH}\end{array}$ & $\mathrm{pH}$ & $\begin{array}{c}\text { Concentration } \\
\text { of phenol } \\
{[\mathrm{PhOH}]}\end{array}$ & $\begin{array}{c}\text { [PhOH] }]_{0} \\
\text { minus } \\
\text { column } 3\end{array}$ & $\begin{array}{c}\text { activity } \\
\{\mathrm{OH}]^{*}\end{array}$ & $\begin{array}{c}\text { Columns }(3+5) \\
\text { divided by } \\
\text { columns } \\
(4-5)\end{array}$ & $\begin{array}{c}\text { log of } \\
\text { Column } \\
6\end{array}$ & $\begin{array}{c}\mathrm{p} K_{\mathrm{a}} \\
(\mathrm{columns} \\
2+7)\end{array}$ \\
\hline 0.00 & 6.98 & 0.0148 & 0.000 & & & & \\
0.25 & 9.25 & 0.0142 & 0.001 & $1.20 \mathrm{E}-05$ & 27.42 & 1.44 & 10.69 \\
0.50 & 9.59 & 0.0136 & 0.001 & $2.63 \mathrm{E}-05$ & 13.24 & 1.12 & 10.71 \\
0.75 & 9.77 & 0.0130 & 0.002 & $3.98 \mathrm{E}-05$ & 8.50 & 0.93 & 10.70 \\
1.00 & 9.90 & 0.0124 & 0.002 & $5.37 \mathrm{E}-05$ & 6.13 & 0.79 & 10.69 \\
1.25 & 10.02 & 0.0118 & 0.003 & $7.08 \mathrm{E}-05$ & 4.71 & 0.67 & 10.69 \\
1.50 & 10.11 & 0.0112 & 0.003 & $8.71 \mathrm{E}-05$ & 3.76 & 0.58 & 10.69 \\
2.00 & 10.27 & 0.0101 & 0.004 & $1.26 \mathrm{E}-04$ & 2.58 & 0.41 & 10.68 \\
2.50 & 10.39 & 0.0090 & 0.005 & $1.66 \mathrm{E}-04$ & 1.87 & 0.27 & 10.66 \\
3.00 & 10.51 & 0.0079 & 0.006 & $2.19 \mathrm{E}-04$ & 1.40 & 0.15 & 10.66 \\
3.50 & 10.63 & 0.0068 & 0.007 & $2.88 \mathrm{E}-04$ & 1.07 & 0.03 & 10.66 \\
4.00 & 10.75 & 0.0058 & 0.008 & $3.80 \mathrm{E}-04$ & 0.82 & -0.08 & 10.67 \\
4.50 & 10.85 & 0.0047 & 0.009 & $4.79 \mathrm{E}-04$ & 0.63 & -0.20 & 10.65 \\
5.00 & 10.95 & 0.0037 & 0.010 & $6.03 \mathrm{E}-04$ & 0.48 & -0.32 & 10.63 \\
5.50 & 11.05 & 0.0028 & 0.010 & $7.59 \mathrm{E}-04$ & 0.36 & -0.44 & 10.61 \\
6.00 & 11.15 & 0.0018 & 0.011 & $9.55 \mathrm{E}-04$ & 0.26 & -0.58 & 10.57 \\
6.50 & 11.28 & 0.0008 & 0.012 & $1.29 \mathrm{E}-03$ & 0.19 & -0.71 & 10.57 \\
\hline & & & & & & &
\end{tabular}

Result: $\mathbf{p} K_{\mathrm{a}}=10.66 \pm 0.08$ at $0.015 \mathrm{M}$ and $25^{\circ} \mathrm{C}$ 
Table S29. Determination of ionization constant of 4-Me-2-MeO-phenol (82.1 mg, $0.594 \mathrm{mmol})$ dissolved in $48 \mathrm{~mL}$ of methanol-water $(1: 1, \mathrm{v} / \mathrm{v}), 25^{\circ} \mathrm{C}$.

\begin{tabular}{|c|c|c|c|c|c|c|c|}
\hline 1 & 2 & 3 & 4 & 5 & 6 & 7 & 8 \\
\hline $\begin{array}{c}\text { Titrant } \\
0.0675 \mathrm{M} \\
\mathrm{KOH}\end{array}$ & $\mathrm{pH}$ & $\begin{array}{c}\text { Concentration } \\
\text { of phenol } \\
{[\mathrm{PhOH}]}\end{array}$ & $\begin{array}{c}\mathrm{PhOH}]_{0} \\
\text { minus } \\
\text { column } 3\end{array}$ & $\begin{array}{l}\text { activity } \\
\{\mathrm{OH}\}^{*}\end{array}$ & $\begin{array}{c}\text { Columns }(3+5) \\
\text { divided by } \\
\text { columns } \\
(4-5)\end{array}$ & $\begin{array}{c}\log \text { of } \\
\text { Column } \\
6\end{array}$ & $\begin{array}{c}\mathrm{p} K_{\mathrm{a}} \\
\text { (columns } \\
2+7 \text { ) }\end{array}$ \\
\hline$(\mathrm{mL})$ & & $M$ & M & & & & \\
\hline 0.00 & 5.23 & 0.0124 & 0.000 & & & & \\
\hline 0.50 & 9.52 & 0.0116 & 0.001 & 2.24E-05 & 17.19 & 1.24 & 10.76 \\
\hline 1.00 & 9.83 & 0.0107 & 0.001 & 4.57E-05 & 8.11 & 0.91 & 10.74 \\
\hline 1.50 & 10.08 & 0.0100 & 0.002 & 8.13E-05 & 5.11 & 0.71 & 10.79 \\
\hline 2.00 & 10.24 & 0.0092 & 0.003 & 1.17E-04 & 3.60 & 0.56 & 10.80 \\
\hline 2.50 & 10.35 & 0.0084 & 0.003 & $1.51 \mathrm{E}-04$ & 2.69 & 0.43 & 10.78 \\
\hline 3.00 & 10.46 & 0.0077 & 0.004 & $1.95 \mathrm{E}-04$ & 2.09 & 0.32 & 10.78 \\
\hline 3.50 & 10.63 & 0.0070 & 0.005 & 2.88E-04 & 1.68 & 0.23 & 10.86 \\
\hline 4.00 & 10.67 & 0.0062 & 0.005 & 3.16E-04 & 1.34 & 0.13 & 10.80 \\
\hline 4.50 & 10.77 & 0.0055 & 0.006 & 3.98E-04 & 1.10 & 0.04 & 10.81 \\
\hline 5.00 & 10.86 & 0.0048 & 0.006 & $4.90 \mathrm{E}-04$ & 0.91 & -0.04 & 10.82 \\
\hline 5.50 & 10.93 & 0.0042 & 0.007 & 5.75E-04 & 0.75 & -0.13 & 10.80 \\
\hline 6.00 & 11.00 & 0.0035 & 0.008 & 6.76E-04 & 0.61 & -0.21 & 10.79 \\
\hline
\end{tabular}

Result: $\mathrm{p} K_{\mathrm{a}}=10.79 \pm 0.07$ at $0.0124 \mathrm{M}$ and $25^{\circ} \mathrm{C}$ 
Table S30. Determination of ionization constant of 4-Me-2-MeO-phenol (89.5 mg, $0.648 \mathrm{mmol})$ dissolved in $45 \mathrm{~mL}$ of methanol-water $(1: 1, \mathrm{v} / \mathrm{v}), 25^{\circ} \mathrm{C}$.

\begin{tabular}{|c|c|c|c|c|c|c|c|}
\hline 1 & 2 & 3 & 4 & 5 & 6 & 7 & 8 \\
\hline $\begin{array}{c}\text { Titrant } \\
0.0675 \mathrm{M} \\
\mathrm{KOH}\end{array}$ & $\mathrm{pH}$ & $\begin{array}{l}\text { Concentration } \\
\text { of phenol } \\
\text { [PhOH] }\end{array}$ & $\begin{array}{c}{[\mathrm{PhOH}]_{\circ}} \\
\text { minus } \\
\text { column } 3\end{array}$ & $\begin{array}{l}\text { activity } \\
\{\mathrm{OH}\}^{*}\end{array}$ & $\begin{array}{c}\text { Columns }(3+5) \\
\text { divided by } \\
\text { columns } \\
(4-5)\end{array}$ & $\begin{array}{l}\log \text { of } \\
\text { Column } \\
\quad 6\end{array}$ & $\begin{array}{c}\mathrm{p} K_{\mathrm{a}} \\
\text { (columns } \\
2+7 \text { ) }\end{array}$ \\
\hline$(\mathrm{mL})$ & & $\mathrm{M}$ & $\mathrm{M}$ & & & & \\
\hline 0.00 & 6.55 & 0.0144 & 0.000 & & & & \\
\hline 0.50 & 9.35 & 0.0135 & 0.001 & $1.51 \mathrm{E}-05$ & 18.59 & 1.27 & 10.62 \\
\hline 1.00 & 9.70 & 0.0126 & 0.001 & 3.39E-05 & 8.82 & 0.95 & 10.65 \\
\hline 1.50 & 9.91 & 0.0118 & 0.002 & 5.50E-05 & 5.56 & 0.75 & 10.66 \\
\hline 2.00 & 10.07 & 0.0109 & 0.003 & 7.94E-05 & 3.93 & 0.59 & 10.66 \\
\hline 2.50 & 10.20 & 0.0101 & 0.004 & 1.07E-04 & 2.96 & 0.47 & 10.67 \\
\hline 3.00 & 10.32 & 0.0093 & 0.004 & $1.41 \mathrm{E}-04$ & 2.31 & 0.36 & 10.68 \\
\hline 3.50 & 10.44 & 0.0085 & 0.005 & $1.86 \mathrm{E}-04$ & 1.85 & 0.27 & 10.71 \\
\hline 4.00 & 10.54 & 0.0077 & 0.006 & 2.34E-04 & 1.51 & 0.18 & 10.72 \\
\hline 4.50 & 10.65 & 0.0069 & 0.006 & 3.02E-04 & 1.24 & 0.09 & 10.74 \\
\hline 5.00 & 10.76 & 0.0062 & 0.007 & 3.89E-04 & 1.04 & 0.02 & 10.78 \\
\hline 5.50 & 10.87 & 0.0055 & 0.007 & $5.01 \mathrm{E}-04$ & 0.87 & -0.06 & 10.81 \\
\hline 6.00 & 10.98 & 0.0048 & 0.008 & 6.46E-04 & 0.74 & -0.13 & 10.85 \\
\hline 6.50 & 11.08 & 0.0041 & 0.009 & 8.13E-04 & 0.63 & -0.20 & 10.88 \\
\hline 7.00 & 11.18 & 0.0034 & 0.009 & $1.02 \mathrm{E}-03$ & 0.54 & -0.26 & 10.92 \\
\hline 7.50 & 11.26 & 0.0027 & 0.010 & $1.23 \mathrm{E}-03$ & 0.47 & -0.33 & 10.93 \\
\hline 8.00 & 11.33 & 0.0020 & 0.010 & $1.45 \mathrm{E}-03$ & 0.40 & -0.40 & 10.93 \\
\hline 8.50 & 11.40 & 0.0014 & 0.011 & 1.70E-03 & 0.34 & -0.47 & 10.93 \\
\hline 9.00 & 11.45 & 0.0007 & 0.011 & 1.91E-03 & 0.28 & -0.55 & 10.90 \\
\hline
\end{tabular}

Result: $\mathrm{p} K_{\mathrm{a}}=\mathbf{1 0 . 7 7} \pm \mathbf{0 . 1 6}$ at $0.0144 \mathrm{M}$ and $25^{\circ} \mathrm{C}$ 
Table S31. Determination of ionization constant of isoeugenol $(92.6 \mathrm{mg}, 0.564 \mathrm{mmol})$ dissolved in $49 \mathrm{~mL}$ of methanol-water $(1: 1, \mathrm{v} / \mathrm{v}), 25^{\circ} \mathrm{C}$.

\begin{tabular}{cccccccc}
\hline $\mathbf{1}$ & $\mathbf{2}$ & $\mathbf{3}$ & $\mathbf{4}$ & $\mathbf{5}$ & $\mathbf{6}$ & $\mathbf{7}$ & $\mathbf{8}$ \\
\hline $\begin{array}{c}\text { Titrant } \\
0.0675 \mathrm{M} \\
\mathrm{KOH}\end{array}$ & $\mathrm{pH}$ & $\begin{array}{c}\text { Concentration } \\
\text { of phenol } \\
\text { [PhOH] } \\
\mathrm{mL})\end{array}$ & $\begin{array}{c}\text { [PhOH] } \\
\text { minus } \\
\text { column } 3 \\
\mathrm{M}\end{array}$ & $\begin{array}{c}\text { activity } \\
\{\mathrm{OH}\}^{*}\end{array}$ & $\begin{array}{c}\text { Columns }(3+5) \\
\text { divided by } \\
\text { columns }(4-5)\end{array}$ & $\begin{array}{c}\text { log of } \\
\text { Column } 6\end{array}$ & $\begin{array}{c}\mathrm{p} K_{\mathrm{a}} \\
\text { (columns } \\
2+7)\end{array}$ \\
\hline 0.00 & 5.69 & 0.0115 & 0.000 & & & & \\
0.50 & 9.13 & 0.0107 & 0.001 & $9.12 \mathrm{E}-06$ & 15.94 & 1.20 & 10.33 \\
1.00 & 9.53 & 0.0099 & 0.001 & $2.29 \mathrm{E}-05$ & 7.50 & 0.88 & 10.41 \\
1.50 & 9.82 & 0.0092 & 0.002 & $4.47 \mathrm{E}-05$ & 4.70 & 0.67 & 10.49 \\
2.00 & 10.00 & 0.0084 & 0.003 & $6.76 \mathrm{E}-05$ & 3.29 & 0.52 & 10.52 \\
2.50 & 10.18 & 0.0077 & 0.003 & $1.02 \mathrm{E}-04$ & 2.45 & 0.39 & 10.57 \\
3.00 & 10.31 & 0.0070 & 0.004 & $1.38 \mathrm{E}-04$ & 1.89 & 0.28 & 10.59 \\
3.50 & 10.44 & 0.0062 & 0.005 & $1.86 \mathrm{E}-04$ & 1.49 & 0.17 & 10.61 \\
4.00 & 10.57 & 0.0055 & 0.005 & $2.51 \mathrm{E}-04$ & 1.20 & 0.08 & 10.65 \\
4.50 & 10.70 & 0.0049 & 0.006 & $3.39 \mathrm{E}-04$ & 0.97 & -0.01 & 10.69 \\
5.00 & 10.82 & 0.0042 & 0.006 & $4.47 \mathrm{E}-04$ & 0.80 & -0.10 & 10.72 \\
5.50 & 10.92 & 0.0035 & 0.007 & $5.62 \mathrm{E}-04$ & 0.66 & -0.18 & 10.74 \\
6.00 & 11.00 & 0.0029 & 0.007 & $6.76 \mathrm{E}-04$ & 0.53 & -0.27 & 10.73 \\
\hline
\end{tabular}

Result: $\mathrm{p} K_{\mathrm{a}}=\mathbf{1 0 . 6 0} \pm \mathbf{0 . 2 5}$ at $0.0115 \mathrm{M}$ and $25^{\circ} \mathrm{C}$

Table S32. Determination of ionization constant of isoeugenol $(92.7 \mathrm{mg}, 0.565 \mathrm{mmol})$ dissolved in $45 \mathrm{~mL}$ of methanol-water $(1: 1, \mathrm{v} / \mathrm{v}), 25^{\circ} \mathrm{C}$.

\begin{tabular}{cccccccc}
\hline $\mathbf{1}$ & $\mathbf{2}$ & $\mathbf{3}$ & $\mathbf{4}$ & $\mathbf{5}$ & $\mathbf{6}$ & $\mathbf{7}$ & $\mathbf{8}$ \\
\hline $\begin{array}{c}\text { Titrant } \\
.0675 \mathrm{M} \\
\mathrm{KOH}\end{array}$ & $\mathrm{pH}$ & $\begin{array}{c}\text { Concentration } \\
\text { of phenol } \\
\text { [PhOH] } \\
(\mathrm{mL})\end{array}$ & $\begin{array}{c}\text { M } \\
\text { minus }]_{0} \\
\text { column } 3 \\
\mathrm{M}\end{array}$ & $\begin{array}{c}\text { activity } \\
\{\mathrm{OH}]^{*}\end{array}$ & $\begin{array}{c}\text { Columns }(3+5) \\
\text { divided by } \\
\text { columns }(4-5)\end{array}$ & $\begin{array}{c}\text { log of } \\
\text { Column } \\
6\end{array}$ & $\begin{array}{c}\mathrm{p} K_{\mathrm{a}} \\
\text { (columns } \\
2+7)\end{array}$ \\
\hline 0.00 & 6.55 & 0.0125 & 0.000 & & & & \\
0.50 & 9.35 & 0.0117 & 0.001 & $1.51 \mathrm{E}-05$ & 16.08 & 1.21 & 10.56 \\
1.00 & 9.70 & 0.0108 & 0.001 & $3.39 \mathrm{E}-05$ & 7.56 & 0.88 & 10.58 \\
1.50 & 9.91 & 0.0100 & 0.002 & $5.50 \mathrm{E}-05$ & 4.72 & 0.67 & 10.58 \\
2.00 & 10.07 & 0.0091 & 0.003 & $7.94 \mathrm{E}-05$ & 3.30 & 0.52 & 10.59 \\
2.50 & 10.20 & 0.0083 & 0.004 & $1.07 \mathrm{E}-04$ & 2.45 & 0.39 & 10.59 \\
3.00 & 10.31 & 0.0075 & 0.004 & $1.38 \mathrm{E}-04$ & 1.88 & 0.27 & 10.58 \\
3.50 & 10.44 & 0.0068 & 0.005 & $1.86 \mathrm{E}-04$ & 1.48 & 0.17 & 10.61 \\
4.00 & 10.57 & 0.0060 & 0.006 & $2.51 \mathrm{E}-04$ & 1.19 & 0.08 & 10.65 \\
4.50 & 10.70 & 0.0053 & 0.006 & $3.39 \mathrm{E}-04$ & 0.97 & -0.01 & 10.69 \\
5.00 & 10.82 & 0.0045 & 0.007 & $4.47 \mathrm{E}-04$ & 0.79 & -0.10 & 10.72 \\
5.50 & 10.92 & 0.0038 & 0.007 & $5.62 \mathrm{E}-04$ & 0.65 & -0.19 & 10.73 \\
6.00 & 11.00 & 0.0031 & 0.008 & $6.76 \mathrm{E}-04$ & 0.52 & -0.28 & 10.72 \\
\hline
\end{tabular}

Result: $\mathrm{p} K_{\mathrm{a}}=10.63 \pm 0.10$ at $0.0125 \mathrm{M}$ and $25^{\circ} \mathrm{C}$ 
Table S33. Determination of ionization constant of $\mathrm{DHZ}(91.0 \mathrm{mg}, 0.473 \mathrm{mmol})$ dissolved in $48.5 \mathrm{~mL}$ of methanol-water $(1: 1, \mathrm{v} / \mathrm{v}), 25^{\circ} \mathrm{C}$.

\begin{tabular}{|c|c|c|c|c|c|c|c|}
\hline 1 & 2 & 3 & 4 & 5 & 6 & 7 & 8 \\
\hline $\begin{array}{c}\text { Titrant } \\
0.0675 \mathrm{M} \\
\mathrm{KOH}\end{array}$ & $\mathrm{pH}$ & $\begin{array}{c}\text { Concentration } \\
\text { of phenol } \\
{[\mathrm{PhOH}]}\end{array}$ & $\begin{array}{c}\mathrm{PhOH}]_{0} \\
\text { minus } \\
\text { column } 3\end{array}$ & $\begin{array}{l}\text { activity } \\
\{\mathrm{OH}\}^{*}\end{array}$ & $\begin{array}{c}\text { Columns }(3+5) \\
\text { divided by } \\
\text { columns } \\
(4-5)\end{array}$ & $\begin{array}{c}\log \text { of } \\
\text { Column } \\
6\end{array}$ & $\begin{array}{c}\mathrm{p} K_{\mathrm{a}} \\
\text { (columns } \\
2+7)\end{array}$ \\
\hline$(\mathrm{mL})$ & & M & M & & & & \\
\hline 0.00 & 5.54 & 0.0098 & 0.000 & & & & \\
\hline 0.25 & 7.45 & 0.0094 & 0.000 & $1.91 \mathrm{E}-07$ & 27.07 & 1.43 & 8.88 \\
\hline 0.50 & 7.8 & 0.0090 & 0.001 & 4.27E-07 & 13.04 & 1.12 & 8.92 \\
\hline 0.75 & 7.99 & 0.0086 & 0.001 & $6.61 \mathrm{E}-07$ & 8.36 & 0.92 & 8.91 \\
\hline 1.00 & 8.16 & 0.0082 & 0.001 & 9.77E-07 & 6.02 & 0.78 & 8.94 \\
\hline 1.25 & 8.26 & 0.0078 & 0.002 & $1.23 \mathrm{E}-06$ & 4.62 & 0.66 & 8.92 \\
\hline 1.50 & 8.44 & 0.0074 & 0.002 & $1.86 \mathrm{E}-06$ & 3.68 & 0.57 & 9.01 \\
\hline 1.75 & 8.52 & 0.0071 & 0.002 & 2.24E-06 & 3.01 & 0.48 & 9.00 \\
\hline 2.00 & 8.63 & 0.0067 & 0.003 & 2.88E-06 & 2.51 & 0.40 & 9.03 \\
\hline 2.25 & 8.71 & 0.0063 & 0.003 & 3.47E-06 & 2.12 & 0.33 & 9.04 \\
\hline 2.50 & 8.81 & 0.0060 & 0.003 & 4.37E-06 & 1.81 & 0.26 & 9.07 \\
\hline 2.75 & 8.89 & 0.0056 & 0.004 & 5.25E-06 & 1.55 & 0.19 & 9.08 \\
\hline 3.00 & 8.97 & 0.0053 & 0.004 & 6.31E-06 & 1.34 & 0.13 & 9.10 \\
\hline 3.25 & 9.04 & 0.0049 & 0.004 & 7.41E-06 & 1.16 & 0.07 & 9.11 \\
\hline 3.50 & 9.12 & 0.0046 & 0.005 & 8.91E-06 & 1.01 & 0.00 & 9.12 \\
\hline 3.75 & 9.21 & 0.0042 & 0.005 & 1.10E-05 & 0.87 & -0.06 & 9.15 \\
\hline 4.00 & 9.28 & 0.0039 & 0.005 & $1.29 \mathrm{E}-05$ & 0.76 & -0.12 & 9.16 \\
\hline 4.25 & 9.43 & 0.0035 & 0.005 & $1.82 \mathrm{E}-05$ & 0.66 & -0.18 & 9.25 \\
\hline 4.50 & 9.5 & 0.0032 & 0.006 & 2.14E-05 & 0.56 & -0.25 & 9.25 \\
\hline 4.75 & 9.62 & 0.0029 & 0.006 & 2.82E-05 & 0.48 & -0.32 & 9.30 \\
\hline
\end{tabular}

Result: $\mathrm{p} K_{\mathrm{a}}=9.1 \pm 0.2$ at $0.010 \mathrm{M}$ and $25^{\circ} \mathrm{C}$ 
Table S34. Determination of ionization constant of $\mathrm{DHZ}(75.8 \mathrm{mg}, 0.394 \mathrm{mmol})$ dissolved in $46 \mathrm{~mL}$ of methanol-water $(1: 1, \mathrm{v} / \mathrm{v}), 25^{\circ} \mathrm{C}$.

\begin{tabular}{|c|c|c|c|c|c|c|c|}
\hline 1 & 2 & 3 & 4 & 5 & 6 & 7 & 8 \\
\hline $\begin{array}{c}\text { Titrant } \\
0.0675 \mathrm{M} \\
\mathrm{KOH}\end{array}$ & $\mathrm{pH}$ & $\begin{array}{c}\text { Concentration } \\
\text { of phenol } \\
{[\mathrm{PhOH}]}\end{array}$ & $\begin{array}{c}{[\mathrm{PhOH}]_{\circ}} \\
\text { minus } \\
\text { column } 3\end{array}$ & $\begin{array}{l}\text { activity } \\
\{\mathrm{OH}\}^{*}\end{array}$ & $\begin{array}{c}\text { Columns }(3+5) \\
\text { divided by } \\
\text { columns } \\
(4-5)\end{array}$ & $\begin{array}{c}\log \text { of } \\
\text { Column } \\
6\end{array}$ & $\begin{array}{c}\mathrm{p} K_{\mathrm{a}} \\
\text { (columns } \\
2+7 \text { ) }\end{array}$ \\
\hline$(\mathrm{mL})$ & & $\mathrm{M}$ & $\mathrm{M}$ & & & & \\
\hline 0.00 & 6.27 & 0.0086 & 0.000 & & & & \\
\hline 0.50 & 8.07 & 0.0078 & 0.001 & 7.94E-07 & 10.70 & 1.03 & 9.10 \\
\hline 1.00 & 8.44 & 0.0070 & 0.001 & 1.86E-06 & 4.85 & 0.69 & 9.13 \\
\hline 1.50 & 8.67 & 0.0062 & 0.002 & 3.16E-06 & 2.90 & 0.46 & 9.13 \\
\hline 2.00 & 8.86 & 0.0054 & 0.003 & 4.90E-06 & 1.93 & 0.28 & 9.14 \\
\hline 2.50 & 9.03 & 0.0047 & 0.003 & 7.24E-06 & 1.34 & 0.13 & 9.16 \\
\hline 3.00 & 9.23 & 0.0039 & 0.004 & 1.15E-05 & 0.95 & -0.02 & 9.21 \\
\hline 3.50 & 9.44 & 0.0032 & 0.005 & 1.86E-05 & 0.68 & -0.17 & 9.27 \\
\hline 4.00 & 9.73 & 0.0025 & 0.005 & 3.63E-05 & 0.47 & -0.33 & 9.40 \\
\hline 4.50 & 9.90 & 0.0018 & 0.006 & 5.37E-05 & 0.31 & -0.51 & 9.39 \\
\hline
\end{tabular}

Result: $\mathbf{p} K_{\mathrm{a}}=\mathbf{9 . 2 0} \pm \mathbf{0 . 2 0}$ at $0.009 \mathrm{M}$ and $25^{\circ} \mathrm{C}$

Table S35. Determination of ionization constant of $\mathrm{DHZ}(73.6 \mathrm{mg}, 0.383 \mathrm{mmol})$ dissolved in $47 \mathrm{~mL}$ of methanol-water $(1: 1, \mathrm{v} / \mathrm{v}), 25^{\circ} \mathrm{C}$.

\begin{tabular}{|c|c|c|c|c|c|c|c|}
\hline 1 & 2 & 3 & 4 & 5 & 6 & 7 & 8 \\
\hline $\begin{array}{c}\text { Titrant } \\
0.100 \mathrm{M} \mathrm{KOH}\end{array}$ & $\mathrm{pH}$ & $\begin{array}{c}\text { Concentration } \\
\text { of phenol } \\
{[\mathrm{PhOH}]}\end{array}$ & $\begin{array}{c}{[\mathrm{PhOH}]_{0}} \\
\text { minus } \\
\text { column } 3\end{array}$ & $\begin{array}{l}\text { activity } \\
\{\mathrm{OH}\}^{*}\end{array}$ & $\begin{array}{c}\text { Columns }(3+5) \\
\text { divided by } \\
\text { columns } \\
(4-5)\end{array}$ & $\begin{array}{c}\log \text { of } \\
\text { Column } \\
6\end{array}$ & $\begin{array}{c}\mathrm{p} K_{\mathrm{a}} \\
\text { (columns } \\
2+7 \text { ) }\end{array}$ \\
\hline$(\mathrm{mL})$ & & $M$ & M & & & & \\
\hline 0.00 & 6.45 & 0.0081 & 0.000 & & & & \\
\hline 0.25 & 7.92 & 0.0076 & 0.001 & 5.62E-07 & 14.33 & 1.16 & 9.08 \\
\hline 0.50 & 8.24 & 0.0070 & 0.001 & 1.17E-06 & 6.67 & 0.82 & 9.06 \\
\hline 0.75 & 8.47 & 0.0064 & 0.002 & $2.00 \mathrm{E}-06$ & 4.11 & 0.61 & 9.08 \\
\hline 1.00 & 8.61 & 0.0059 & 0.002 & $2.75 \mathrm{E}-06$ & 2.83 & 0.45 & 9.06 \\
\hline 1.25 & 8.74 & 0.0053 & 0.003 & 3.72E-06 & 2.07 & 0.32 & 9.06 \\
\hline 1.50 & 8.89 & 0.0048 & 0.003 & 5.25E-06 & 1.56 & 0.19 & 9.08 \\
\hline 1.75 & 9.01 & 0.0043 & 0.004 & 6.92E-06 & 1.19 & 0.08 & 9.09 \\
\hline 2.00 & 9.13 & 0.0037 & 0.004 & $9.12 \mathrm{E}-06$ & 0.92 & -0.04 & 9.09 \\
\hline 2.25 & 9.27 & 0.0032 & 0.005 & $1.26 \mathrm{E}-05$ & 0.71 & -0.15 & 9.12 \\
\hline 2.50 & 9.4 & 0.0027 & 0.005 & 1.70E-05 & 0.54 & -0.27 & 9.13 \\
\hline 2.75 & 9.55 & 0.0022 & 0.006 & $2.40 \mathrm{E}-05$ & 0.40 & -0.40 & 9.15 \\
\hline 3.00 & 9.79 & 0.0017 & 0.006 & 4.17E-05 & 0.29 & -0.54 & 9.25 \\
\hline 3.25 & 9.95 & 0.0012 & 0.006 & 6.03E-05 & 0.19 & -0.72 & 9.23 \\
\hline
\end{tabular}

Result: $\mathrm{p} K_{\mathrm{a}}=9.11 \pm 0.12$ at $0.008 \mathrm{M}$ and $25^{\circ} \mathrm{C}$ 
Table S36. Experimental and literature values of $\mathrm{p} K_{\mathrm{a}}$ for the studied phenols.

\begin{tabular}{|c|c|c|c|c|}
\hline & \multirow{2}{*}{$\begin{array}{c}\text { This work } \\
\mathrm{H}_{2} \mathrm{O}: \text { methanol } \\
\text { 50:50 v/v } \\
\end{array}$} & \multicolumn{3}{|c|}{ Literature } \\
\hline & & $\begin{array}{c}\mathrm{H}_{2} \mathrm{O}: \text { methanol } \\
50: 50 \mathrm{v} / \mathrm{v}\end{array}$ & $\mathrm{H}_{2} \mathrm{O}^{a}$ & methanol \\
\hline 2-MeO-phenol & $\begin{array}{l}10.66 \pm 0.09 \\
10.69 \pm 0.08\end{array}$ & $11.03^{b}$ & $\begin{array}{l}9.85 \\
9.90 \\
9.98 \\
9.94^{b}\end{array}$ & $14.48^{C}$ \\
\hline 4-Me-2-MeO-phenol & $\begin{array}{l}10.77 \pm 0.16 \\
10.79 \pm 0.07\end{array}$ & & $\begin{array}{l}10.21 \\
10.28\end{array}$ & \\
\hline IE & $\begin{array}{l}10.60 \pm 0.10 \\
10.60 \pm 0.20\end{array}$ & & $\begin{array}{l}9.88 \\
9.89 \\
9.5^{d}\end{array}$ & \\
\hline DHZ & $\begin{array}{l}9.20 \pm 0.20 \\
9.11 \pm 0.15 \\
9.05 \pm 0.22\end{array}$ & & $8.3^{d}$ & \\
\hline Curcumin & & $\begin{array}{l}\mathrm{p} K \mathrm{Ka}(1)=8.54^{e} \\
\mathrm{p} K_{\mathrm{a}}(2)=9.30^{e} \\
\mathrm{p} K_{\mathrm{a}}(3)=10.69^{e}\end{array}$ & $\begin{array}{l}\mathrm{p} K \mathrm{~K}(1)=7.75^{f} \\
\mathrm{p} K_{\mathrm{a}}(2)=8.55^{f} \\
\mathrm{p} K_{\mathrm{a}}(3)=9.05^{f}\end{array}$ & $\mathrm{p} K_{\mathrm{a}}(1)=12.1^{e}$ \\
\hline Dimethoxycurcumin ${ }^{g}$ & & $8.75^{e}$ & & $12.9^{e}$ \\
\hline
\end{tabular}

${ }^{a}$ From Sergeant, E. P.; Dempsey, B.: Ionization constants of organic acids in aqueous solution. IUPAC Chemical Data Series - No. 23, Pergamon Press, Oxford 1979.

${ }^{b}$ Roses, M.; Rived, F.; Bosch, E. J. Chromatogr. A 2000, 867, 45-56.

${ }^{c}$ Rived, F.; Roses, M.; Bosch, E. Anal. Chim. Acta 1998, 374, 309-324.

${ }^{d}$ Values probably mainly in water (for IE - in water+ $10 \%$ acetonitrile), Priyadarsini, K. I.; Guha, S. N.; Rao, M. N. A. Free Rad. Biol. Med. 1998, 24, 933-941.

${ }^{e}$ Borsari M.; Ferrari, E.; Grandi, R.; Saladini M.; Inorg. Chim. Acta 2002, 328, 61-68; measured by potentiometric, spectrophotometric and NMR methods.

${ }^{f}$ Tønnesen, H. H.; Karlsen, J. Z.Lebensm. Unters. Forsch. 1985, 180, 402-404.

${ }^{g}$ The dimethyl ether of $\mathrm{CU}$ having no phenolic $\mathrm{OH}$ groups. 